\title{
Transcriptional Targeting of Mature Dendritic Cells with Adenoviral Vectors via a Modular Promoter System for Antigen Expression and Functional Manipulation
}

\author{
Ilka Knippertz, ${ }^{1}$ Andrea Deinzer, ${ }^{1}$ Jan Dörrie, ${ }^{2}$ Niels Schaft, ${ }^{2}$ \\ Dirk M. Nettelbeck, ${ }^{3}$ and Alexander Steinkasserer ${ }^{1}$ \\ ${ }^{1}$ Department of Immune Modulation at the Department of Dermatology, Universitätsklinikum Erlangen, Hartmannstrasse 14, \\ 91052 Erlangen, Germany \\ ${ }^{2}$ Department of Dermatology, Universitätsklinikum Erlangen, Hartmannstrasse 14, 91052 Erlangen, Germany \\ ${ }^{3}$ German Cancer Research Center, Im Neuenheimer Feld 280, 69120 Heidelberg, Germany
}

Correspondence should be addressed to Ilka Knippertz; ilka.knippertz@uk-erlangen.de

Received 17 March 2016; Accepted 29 May 2016

Academic Editor: Pablo González

Copyright (C) 2016 Ilka Knippertz et al. This is an open access article distributed under the Creative Commons Attribution License, which permits unrestricted use, distribution, and reproduction in any medium, provided the original work is properly cited.

\begin{abstract}
To specifically target dendritic cells (DCs) to simultaneously express different therapeutic transgenes for inducing immune responses against tumors, we used a combined promoter system of adenoviral vectors. We selected a 216 bp short Hsp70B' core promoter induced by a mutated, constitutively active heat shock factor (mHSF) 1 to drive strong gene expression of therapeutic transgenes MelanA, BclxL, and IL-12p70 in HeLa cells, as well as in mature DCs (mDCs). As this involves overexpressing mHSF1, we first evaluated the resulting effects on DCs regarding upregulation of heat shock proteins and maturation markers, toxicity, cytokine profile, and capacity to induce antigen-specific CD8 ${ }^{+} \mathrm{T}$ cells. Second, we generated the two-vector-based "modular promoter" system, where one vector contains the mHSF1 under the control of the human CD83 promoter, which is specifically active only in DCs and after maturation. mHSF1, in turn, activates the Hsp70B' core promotor-driven expression of transgenes MelanA and IL12 p70 in the DC-like cell line XS52 and in human mature and hence immunogenic DCs, but not in tolerogenic immature DCs. These in vitro experiments provide the basis for an in vivo targeting of mature DCs for the expression of multiple transgenes. Therefore, this modular promoter system represents a promising tool for future DC-based immunotherapies in vivo.
\end{abstract}

\section{Introduction}

Dendritic cells (DCs) function as sentinels at the interface of the innate and the adaptive immune system, thereby inducing highly potent and antigen-specific immune responses triggered by "danger" signals. As master antigen-presenting cells (APCs), they not only express proinflammatory cytokines (particularly IL-12) but also capture, process, and present antigens on major histocompatibility (MHC) molecules to naïve $\mathrm{T}$ cells, leading to an adaptive $\mathrm{T}$ cell-mediated immune response [1]. Importantly, DCs possess the unique feature to cross-present typical MHC class II restricted antigens via MHC class I, thereby inducing cytotoxic $\mathrm{CD} 8^{+}$cell (CTL) responses and enhancing antitumor humoral responses $[2,3]$.
In this context, DCs have been shown to have a significant impact on oncogenesis, tumor progression, and response to therapy in various preclinical tumor models and preclinical studies [4]. Additionally, the discovery of immune checkpoint blockers (e.g., monoclonal antibody [mAb] to CTLA-4; mAb to $\mathrm{PD}-1)$ and their possible combination with $\mathrm{DC}$ vaccination has made cancer immunotherapy one of the most exciting topics in the oncology field recently $[5,6]$. The main DCbased anticancer interventions developed so far comprise various ex vivo and in vivo immune manipulating strategies. Ex vivo, DCs were loaded with different tumor associated antigens (TAAs) using various strategies including (i) peptide or protein pulsing, (ii) loading with complete tumor lysate or (iii) tumor apoptotic bodies, and (iv) RNA transfection 
or (v) viral transduction [7]. In vivo, cancer antigens can be delivered to DCs by fusing them to monoclonal antibodies specifically targeting DC surface receptors such as mannose receptor, C type 1 (MRC1), CD209 (DC-SIGN), or DEC-205 [7-10]. Another strategy is to incorporate TAAs, DNA, RNA, or toll-like receptor ligands into DC-targeting immunoliposomes, nanoparticles, or viral vectors for delivery [11-14].

In recent years, viral vectors like lentiviruses or adenoviruses (Ads) have been widely used in clinical trials for many different types of inherited or acquired disorders with Ad vectors being the ones most commonly used for cancer gene therapy $[15,16]$. Adenoviruses have many advantages as they (i) can be grown into high titer stable stocks, (ii) infect nondividing and dividing cells of different types, (iii) are maintained in cells as an episome, and (iv) have been proven safe and well tolerated while also being therapeutically active [16]. Most Ad vectors are genetically modified versions of Ad5 and can be either replication-defective or replication-competent. Replicationdefective Ad vectors are effectively used as gene delivery vehicles and have the essential E1A, E1B, and E3 genes deleted and replaced by an expression cassette encoding for foreign therapeutic transgenes up to $\sim 6.5 \mathrm{~kb}$ in size [16]. Specific targeting of a cell (e.g., a DC) can be achieved by transductional or transcriptional targeting or a combination of both. Transductional targeting involves chemically or genetically modifying an adenovirus to redirect its tropism from its natural binding partner CAR (coxsackie and adenovirus receptor) to a new target expressed preferentially on the target cell like, for example, DC-SIGN or DEC-205 for DCs [17]. Transcriptional targeting, on the other hand, involves using tissue-specific promoters to genetically limit expression of the introduced gene to distinct tissues [17].

However, there was no cell type- and maturation-specific promoter available for DCs until recently. In 2013, we identified and characterized a tripartite promoter complex specifically regulating human CD83 expression in mature immunogenic DCs. We found that it consisted of a $261 \mathrm{bp}$ core promoter flanked by (i) a $164 \mathrm{bp}$ upstream regulatory element and (ii) a $185 \mathrm{bp}$ downstream enhancer separated from the promoter by a $500 \mathrm{bp}$ noncoding spacer sequence [18]. All three elements were shown to be essential for transcriptional activation in mature immunogenic DCs, while not mediating this specific activation in tolerogenic immature DCs (iDCs), or other CD83-positive cell types, such as subsets of activated $\mathrm{B}$ or $\mathrm{T}$ cells. Thus, this promoter complex shows great potential for the transcriptional targeting of mature DCs and thereby the development of new immunotherapeutic approaches.

Despite the successes of DC-based immunotherapy in individual patients, the immunogenic potential to induce effective antitumor CTL responses is still considered suboptimal [19]. Furthermore, the ex vivo generation of DC-vaccines is laborious and expensive. Hence, new vaccination strategies involving in vivo targeting of DCs for antigen expression and functional manipulation should be addressed. To do this, we developed a combined promoter system to transcriptionally target human DCs to express several therapeutic transgenes at the same time, the "modular promoter (MP)" system. Due to the limited space for foreign DNA in adenoviral vectors, it is problematic to use large, cell-specific promoters for several transgenes. Therefore, we combined the cell type- and maturation-specific CD83 promoter, which has a size of $1.2 \mathrm{~kb}$ [18], with another short and induction-specific promoter in a two-vector system. In this system, the transgenes in one vector are under the control of a short inducible promoter, which is activated by a factor, expressed from the larger, highly specific CD83 promotor in the second vector. As a short, inducible promoter we chose the short heat shock protein (Hsp) 70B' promoter, which has been reported before to mediate specifically heat-dependent transgene expression in replication-deficient adenoviruses [20]. The $h s p 70 B^{\prime}$ gene, along with $h s p 70(A)-1, h s p 70(A)-2$, and $h s p 70 B$, belongs to the $h s p 70$ gene family, all regulated by the heat shock transcription factor 1 (HSF1) [20-23]. HSF1 is a highly conserved transcription factor that coordinates stress-induced transcription and directs versatile physiological processes in eukaryotes [24]. Upon induction, it undergoes trimerization, as well as phosphorylation, followed by nuclear translocation and DNA binding to heat shock promoters [25].

For our MP system we used a mutated, constitutively active HSF1 (mHSF1) [26] whose expression is controlled here by the DC- and maturation-specific human CD83 promoter [18]. In turn, mHSF1 then binds to the short heat shock response element $\mathrm{Hsp} 70 \mathrm{~B}^{\prime}$ driving the simultaneous expression of multiple therapeutic transgenes. Concomitantly, mHSF1 also binds to endogenous heat shock promoters of targeted DCs. We have shown previously that exposure of human DCs to thermal stress leads to an upregulation of Hsp70A, costimulatory molecules, and proinflammatory cytokines, as well as a markedly improved capacity to prime autologous naïve $\mathrm{CD} 8^{+} \mathrm{T}$ cells in vitro [27]. Therefore, in the present study we also analyzed the effects of mHSF1 overexpression on DCs.

Our results demonstrate that the newly generated MP system allows, for the first time, specific and simultaneous expression of different therapeutic transgenes in human mature DCs in vitro, representing a promising tool to improve future DC-based immunotherapies. Moreover, we found no effects regarding the viability, maturation, and function of DCs by overexpressing mHSF1.

\section{Methods}

2.1. Human Dendritic Cells and $C D 8^{+} T$ Cells. Generation of human monocyte-derived dendritic cells (DCs) was performed as previously described [28]. In brief, peripheral blood mononuclear cells (PBMCs) were prepared from leukoreduction system chambers (LRSCs) of healthy donors by density centrifugation, followed by plastic adherence on tissue culture dishes (BD Falcon, MA, US). The nonadherent fraction was stored at $-80^{\circ} \mathrm{C}$ for isolation of autologous $\mathrm{CD}^{+} \mathrm{T}$ cells, while the adherent cell fraction was cultured for 4 days in DC-medium consisting of RPMI 1640 (Lonza, Basel, Switzerland) supplemented with $1 \%$ (vol/vol) of heat-inactivated human serum type AB (Lonza), 1\% Penicillin/Streptomycin/L-Glutamine (Sigma-Aldrich, St. Louis, MO, US), and $10 \mathrm{mM}$ Hepes (Lonza) as well as 
$800 \mathrm{IU} / \mathrm{mL}$ (day 0) or $400 \mathrm{IU} / \mathrm{mL}$ (day 3) recombinant human granulocyte macrophage colony-stimulating factor (GM$\mathrm{CSF}$ ) and $250 \mathrm{IU} / \mathrm{mL}$ (day 0 and 3) recombinant IL-4 (both Miltenyi, Bergisch-Gladbach, Germany). On day 4, immature DCs (iDCs) were used for further experiments. Maturation of DCs was induced by the addition of a maturation cocktail (MC) consisting of $200 \mathrm{U} / \mathrm{mL}$ IL-1 $\beta, 1000 \mathrm{U} / \mathrm{mL}$ IL-6 (both CellGenix, Freiburg, Germany), $10 \mathrm{ng} / \mathrm{mL}$ TNF- $\alpha$ (Beromun; Boehringer Ingelheim, Germany), and $1 \mu \mathrm{g} / \mathrm{mL} \mathrm{PGE}{ }_{2}$ (Prostin $\mathrm{E}_{2}$, Pfizer, NY, US) for 24 or 48 hours or by $0.1 \mathrm{ng} / \mathrm{mL}$ lipopolysaccharide (LPS; Sigma-Aldrich) for 20 hours. For the isolation of autologous $\mathrm{CD}^{+} \mathrm{T}$ cells the nonadherent fraction was thawed and $\mathrm{CD} 8^{+} \mathrm{T}$ cells were isolated using anti-CD8 MACS beads (Miltenyi) according to the manufacturer's instructions. Afterwards cells were cultured in RPMI 1640 (Lonza) medium additionally containing 10\% (vol/vol) of heat-inactivated human serum type $\mathrm{AB}$ (Lonza), $1 \% \mathrm{~L}$ Glutamine (Sigma-Aldrich), $20 \mathrm{mg} / \mathrm{L}$ gentamycin, $10 \mathrm{mM}$ Hepes (Lonza), $1 \mathrm{mM}$ sodium pyruvate (Lonza), and $1 \%$ MEM nonessential aa (PAN Biotech, Aidenbach, Germany). Cryopreservation and thawing of $\mathrm{CD}^{+} \mathrm{T}$ cells were performed as described elsewhere [29]. Cell counting was performed by using a Neubauer counting chamber and Trypan Blue for exclusion of dead cells. Whenever relevant, HLA$\mathrm{A} 0201^{+}$donor material was used.

2.2. Approvals and Legal Requirements. For the generation of PBMCs, moDCs, and $\mathrm{CD}^{+} \mathrm{T}$ cells from LRSCs of healthy donors, the positive vote from the local ethics committee was obtained (ethics vote number 4556).

2.3. Cell Lines. HeLa cells were cultured in Dulbecco's Modified Eagle Medium (DMEM; Lonza) supplemented with 10\% (vol/vol) FCS (PAA/GE Healthcare, Little Chalfont, UK) and 1\% (vol/vol) Penicillin/Streptomycin/L-Glutamine (SigmaAldrich). XS52 cells, kindly provided by A. Takashima (University of Texas Southwestern Medical Center, Dallas, TX, US), were cultured in Iscove's Modified Dulbecco's Medium (IMDM; Lonza) supplemented with $10 \%$ (vol/vol) FCS, $1 \%$ (vol/vol) Penicillin/Streptomycin/L-Glutamine, 1\% (vol/vol) sodium pyruvate (Lonza), 10\% (vol/vol) NS47 supernatant, and $10 \mathrm{ng} / \mathrm{mL}$ murine GM-CSF. 293 (Quantum) cells were cultured in RPMI 1640 (Lonza) supplemented with $10 \%$ (vol/vol) FCS and 1\% (vol/vol) Penicillin/Streptomycin/LGlutamine.

2.4. Plasmid Vectors. The promoterless pGL3-Basic luciferase reporter vector (Promega, WI, US) was used for determination of vector-related background activity and to generate $\mathrm{pHsp} 7 \mathrm{~B}_{-29 /-489}^{\prime}$ and $\mathrm{pHsp}^{-} 0 \mathrm{~B}_{-29 /-242}^{\prime}$ by digesting the human $h s p 70 B$ gene $5^{\prime}$-region (according to GenBank accession no. X13229) with HindIII/BamHI or HindIII/SmaI, respectively. pHsp70 $\mathrm{B}_{-29 /-242}^{\prime}$ was then used to generate pMelA, pBclxL, and pIL-12 by replacing the luciferase gene by the open reading frame sequences of either MelanA/MART1, Bcl-xL, or the human single-chain of IL-12(p70) [30] (kindly provided by F. Schnieders, Provecs Medical GmbH, Hamburg, Germany). The vector pMelA/BclxL/IL-12 was then generated by the sequential connection of the expression cassettes Hsp70B ${ }_{-29 /-242}^{\prime}$-MelanA/, Hsp70B ${ }_{-29 /-242}$-BclxL/, and Hsp70B ${ }_{-29 /-242}^{\prime}$-IL-12(p70). Plasmids expressing mHSF1 under the control of the human CD83 promoter (P-510) were manufactured by replacing the luciferase gene by the open reading frame sequence of mHSF1 [26] (kindly provided by R. Voellmy, HSF Pharmaceuticals, Fribourg, Switzerland) of pGL3-CD83 promoter constructs described before [18], resulting in pP-510/mHSF1, pEs/P-510/mHSF1, and $\mathrm{pEas} / \mathrm{P}-510 / \mathrm{mHSF} 1$. All constructs were generated by standard cloning procedures.

The pGL3-Promoter vector (Promega), containing a SV40 promoter, was used as a positive control and to determine transfection efficacy.

All plasmids for transient transfection experiments were purified by standard endo-free anion-exchange columns (Qiagen, Hilden, Germany) and verified by DNA sequencing (MWG Biotech, Ebersberg, Germany).

2.5. Recombinant Adenoviruses. Ad5MelA/BclxL/IL-12, Ad5MP2, Ad5mHSF1, Ad5P-510/mHSF1, Ad5Es/P-510/mHSF1, Ad5Eas/P-510/mHSF1, Ad5MelA, Ad5Lucl, and Ad5TL are first generation, E1- and E3-deleted, replication-deficient adenoviral vectors. Ad5mHSF1 contains mHSF1 [26] under the control of a CMV promoter, kindly provided by $\mathrm{R}$. Voellmy (HSF Pharmaceuticals, Fribourg, Switzerland). Ad5Lucl contains a CMV-firefly luciferase cassette and Ad5TL contains both a CMV-firefly luciferase cassette and a CMV-GFP cassette (both kindly provided by D. T. Curiel, Washington University School of Medicine, MO, US). All other replication-deficient adenoviruses were cloned as follows: a gene cassette containing either a Hsp70B ${ }_{-29 /-242^{-}}$ MelanA/Hsp70B ${ }_{-29 /-242}$-BclxL/Hsp70B ${ }_{-29 /-242}^{\prime}$-IL-12(p70)-, a Hsp70B ${ }_{-29 /-242}^{\prime}$-MelanA/Hsp70B ${ }_{-29 /-242}^{\prime}$-IL-12(p70)- (MP2), a P-510-mHSF1-, Es/P-510-mHSF1-, Eas/P-510-mHSF1-, or a CMV-MelanA sequence was inserted into pShuttle. Virus genomes were obtained by homologous recombination of the corresponding shuttle plasmids containing the different expression cassettes indicated above with pAdEasy- 1 in E. coli BJ5183 as described before [31]. Adenovirus particles were produced by transfection of the different PacI-digested pAd vectors into 293 cells using Lipofectamine (Invitrogen/Life Technologies, CA, US). All viruses were amplified in 293 cells and purified by two rounds of $\mathrm{CsCl}$ equilibrium density gradient ultracentrifugation. Verification of viral genomes and exclusion of wild-type contamination were performed by PCR. Physical particle concentration [viral particles (vp)/mL] was determined by $\mathrm{OD}_{260}$ reading and infectious particle concentration was determined by TCID $_{50}$ assay on 293 cells.

2.6. Transfection of Cell Lines. For transfection of HeLa cells, $5 \times 10^{4}$ or $8 \times 10^{4}$ cells were seeded in a 24 -well or 12 -well plate (BD Falcon) in a final volume of $500 \mu \mathrm{L}$ or $1 \mathrm{~mL}$ growth medium per well, respectively. The next day, HeLa cells were transfected using Lipofectamine ${ }^{\mathrm{TM}}$ LTX with Plus $^{\mathrm{TM}}$ reagent (Invitrogen/Life Technologies) according to the manufacturer's protocol with a total amount of $0.5 \mu \mathrm{g}$ (24well) or $0.7 \mu \mathrm{g}$ (12-well) DNA. Twenty-four hours later, cells 
were transduced with adenoviruses at $300 \mathrm{TCID}_{50} /$ cell in a final volume of $200 \mu \mathrm{L}$ DMEM (Lonza) containing $2 \%$ FCS (PAA/GE Healthcare). After 1.5 hours of incubation at room temperature on a rocker, $800 \mu \mathrm{L}$ (24-well) or $2 \mathrm{~mL}$ (12-well) growth medium was added. Cells were harvested for further experiments one day after transduction.

For transfection of XS52 cells, $5 \times 10^{5}$ cells were seeded in a 6-well plate (BD Falcon) in a final volume of $2 \mathrm{~mL}$ cell growth medium per well. The following day, cells were transduced using X-tremeGENE ${ }^{\mathrm{TM}}$ HP DNA Transfection Reagent (Roche, Basel, Switzerland) according to the manufacturer's instructions with $2 \mu \mathrm{g}$ DNA and $6 \mu \mathrm{L}$ transfection reagent in a final volume of $200 \mu \mathrm{L}$ Opti-MEM I Reduced Serum Medium (Gibco/ThermoFisher Scientific, MA, US). Four hours after transfection, $2 \mathrm{~mL}$ of growth medium was added and cells were incubated for another 24 hours at $37^{\circ} \mathrm{C} / 5 \% \mathrm{CO}_{2}$ before they were used for further analyses.

2.7. Adenoviral Transduction of Dendritic Cells. Immature $\mathrm{d} 4$ DCs were seeded in 12-well tissue culture plates (BD Falcon) at a concentration of $1 \times 10^{6}$ cells/well in $250 \mu \mathrm{L}$ medium supplemented with either $800 \mathrm{U} / \mathrm{mL}$ GM-CSF and $500 \mathrm{U} / \mathrm{mL}$ IL-4 (for iDCs) or the maturation cocktail consisting of $400 \mathrm{U} / \mathrm{mL}$ IL-1 $\beta, 2000 \mathrm{U} / \mathrm{mL}$ IL-6, $20 \mathrm{ng} / \mathrm{mL}$ TNF- $\alpha$, and $2 \mu \mathrm{g} / \mathrm{mL} \mathrm{PGE}_{2}$, in addition to IL- 4 and GM-CSF (for mDCs). When indicated, $0.1 \mathrm{ng} / \mathrm{mL}$ LPS were used instead of the cytokine cocktail, which was added to the cell culture after the transduction. Adenovirus was added to the cells at 500 $\mathrm{TCID}_{50} /$ cell in a final volume of $250 \mu \mathrm{L}$ medium without cytokines. For cotransduction of two adenoviruses, the final volume was halved to $125 \mu \mathrm{L}$ medium without cytokines, also resulting in a final infection volume of $500 \mu \mathrm{L}$. After 1.5 hours of incubation at room temperature, $2 \mathrm{~mL}$ of growth medium replenished with cytokines as described before was added per well. To determine transduction efficacy, cells were transduced with Ad5TL and the percentage of living green fluorescent cells was assessed by flow cytometric analysis with a FACScan cell analyzer (BD Biosciences, NJ, US). Only experiments that yielded transduction efficiencies of more than $70 \%$ were evaluated and are shown.

\subsection{Transfection and Peptide Pulsing of Dendritic Cells.} Immature DCs were transduced with Ad5Lucl or Ad5mHSF1 or medium-treated in the presence of the cytokine maturation cocktail as described above. The next day, transfection of mature DCs with MelanA/MART-1 RNA was performed using the nonlipid cationic reagent Transmessenger ${ }^{\mathrm{TM}}$ transfection kit (Qiagen) following an adapted protocol from Liao et al. [32] as described previously in detail by Schaft et al. [33]. For peptide pulsing, mature DCs were incubated with $10 \mu \mathrm{g} / \mathrm{mL}$ of the MelanA-derived HLA-A2-binding analogue peptide ELAGIGILTV in DC cell culture medium for 1 hour at $37^{\circ} \mathrm{C} / 5 \% \mathrm{CO}_{2}$.

2.9. Priming and Tetramer Staining of $C D 8^{+} T$ Cells. MelanA RNA-transfected or MelanA peptide-loaded mature DCs were used to stimulate autologous MACS-sorted $\mathrm{CD}^{+} \mathrm{T}$ cells at a ratio of $1: 10$ for one week at $37^{\circ} \mathrm{C} / 5 \% \mathrm{CO}_{2}$. Fresh $\mathrm{T}$ cell medium was added when necessary and on days
2 and $41000 \mathrm{IU} / \mathrm{mL}$ IL-2 (Novartis, Nürnberg, Germany) and $10 \mathrm{ng} / \mathrm{mL}$ IL-7 were supplemented. On day 7, cells were harvested and stained with a HLA-A2-MelanA/iTag MHC class I-tetramer (Beckmann Coulter, Krefeld, Germany) in combination with an anti-CD8-PC7 antibody (Beckmann Coulter). Finally, cells were analyzed using a FC500 cytofluorometer (Beckmann Coulter).

2.10. Flow Cytometric Analyses. Cells were stained with specific $\mathrm{mAb}$ or appropriate isotype controls for $30 \mathrm{~min}$ at $4^{\circ} \mathrm{C}$ in FACS buffer (Dulbecco's PBS [Lonza] containing $2 \%$ FCS [PAA/GE Healthcare]), washed twice, and finally resuspended in cold FACS buffer containing $0.1 \mu \mathrm{g} / \mathrm{mL}$ propidium iodide (PI) (Carl Roth, Karlsruhe, Germany). Stained cells were immediately analyzed with a FACScan cell analyzer (BD Biosciences). Cell debris and dead cells were excluded from the analyses by gating on proper forward and sideward light scatter and on PI negative cells. Also, percentages of PI positive cells were calculated using this gating strategy. A minimum of $10^{4}$ living cells was measured for each sample and results were analyzed using FCS Express 4 Flow Cytometry Software (De Novo Software, CA, US). The following monoclonal antibodies (all from BD Biosciences) were used to determine the phenotype of DCs: PE-labeled mouse anti-human CD25 (M-A251), CD80 (L307.4), CD83 (HB15e), CD86 (IT2.2), HLA-ABC (G46-2.6), and HLA-DR (G46.6). Isotype $\mathrm{mAb}$ controls (all from $\mathrm{BD}$ Biosciences) used but not shown were IgG1-PE (MOPC-21), IgG2a-PE (G155-178), and IgG2b-PE (27-35).

2.11. Cytometric Bead Array. Inflammatory cytokines secreted by DCs into the supernatant were assessed using the Human Inflammatory Cytokine Cytometric Bead Array (CBA; BD Biosciences) according to the manufacturer's protocol.

2.12. ELISA. IL-12p40 and IL-12p70 concentrations in cell culture supernatants were determined by standard two-site sandwich BD OptEIA ${ }^{\mathrm{TM}}$ Human IL-12 (p70) and Human IL-12 (p40) ELISA Kits (BD Biosciences) according to the manufacturer's manual.

2.13. BCA Protein Assay. Protein concentrations of cell lysates for SDS-PAGE and luciferase reporter assays were determined by Pierce ${ }^{\mathrm{TM}}$ Protein Assay Kit (ThermoFisher Scientific, MA, US) according to the manufacturer's protocol.

2.14. SDS-PAGE and Western Blotting. Whole cell extracts for SDS-PAGE were generated as follows: $1 \times 10^{6}$ DCs or XS52 cells were washed with ice-cold PBS and then resuspended in $50 \mu \mathrm{L}$ lysis buffer mixed with $\mathrm{NaVO}_{3}, \mathrm{NaF}$, and PMSF. Afterwards, protein concentrations were determined by the BCA protein assay described above. To prepare sample proteins, they were boiled in the presence of a loading dye mixed with SDS and 2-mercaptoethanol for $10 \mathrm{~min}$ at $95^{\circ} \mathrm{C}$. Then, 20 to $30 \mu \mathrm{g}$ of total protein per sample was separated on a $12.5 \%$ polyacrylamide gel and afterwards transferred onto nitrocellulose filters (Schleicher \& Schüll/GE Healthcare, UK) with a pore size of $0.2 \mu \mathrm{m}$ with the wet blotting 
device "Mini-Protean II Cell and System" (BioRad, CA, US). Membranes were incubated with primary antibodies (Abs) diluted 1:1000-1:100 against HSF1, Hsp40, Hsp70A (C92F3A-5), Hsp70B', Hsp90 (AC-88), Grp94 (9G10) all from Stressgen (distributed by Biomol, Hamburg, Germany), BclxL (2H12; Calbiochem/Merck-Millipore, Darmstadt, Germany), MelanA (A103; Sigma-Aldrich), or beta-actin (AC74; Sigma-Aldrich), followed by HRP-conjugated horse antimouse IgG, goat anti-rabbit IgG, or goat anti-rat IgG Abs (all Cell Signaling Technology, UK) diluted 1:10000-1:2000. Detection was performed with the chemiluminescent Pierce ECL Western Blotting Substrate (ThermoFisher Scientific) on a high performance chemiluminescence film (GE Healthcare).

2.15. Luciferase Reporter Assay. Luciferase activity was measured using the luciferase assay system (Promega) according to the manufacturer's instructions, but utilizing $50 \mu \mathrm{L}$ of luciferase assay reagent and $10 \mu \mathrm{L}$ of cell lysate. Relative luminescence units (RLUs) were obtained with a Wallac Victor plate reader (PerkinElmer, MA, US). RLUs were normalized to the protein concentration as determined using the Pierce BCA $^{\text {TM }}$ Protein Assay Kit (ThermoFisher Scientific).

2.16. Statistical Analyses. The significance of differences was determined by using either one-way ANOVA or two-way ANOVA and Bonferroni's Multiple Comparison post hoc test; $P$ values $<0.05$ were considered statistically significant.

\section{Results}

3.1. The "Modular Promoter" System. We considered two aspects to be important for the development of a combined promoter system, the so-called modular promoter (MP) system, to transcriptionally target DCs. On the one hand, the system should be highly specific for the targeted DC and on the other hand, the therapeutic transgenes should be expressed very efficiently. As there is only limited space for foreign DNA in plasmid and especially viral vectors, we envisioned a dual vector system, in which one vector expresses a mutated, constitutively active heat shock factor (mHSF) 1 [26] under the control of the human mDC-specific CD83 promoter [18] (Figure 1(a)). The mHSF1, in turn, will then induce simultaneous expression of different therapeutic transgenes contained in the second vector, such as tumor antigens, proinflammatory cytokines, or apoptosis inhibitors, by binding to the short heat shock response elements (HRE; Figure 1(a)). We hypothesized that the expression of tumor antigens in combination with different therapeutic proteins will subsequently enhance the capacity of these modified DCs to induce improved and potent antitumoral immune responses.

Of note, mHSF1 may also induce the expression of different endogenous heat shock proteins, such as Hsp70 or Hsp90, by binding to the cellular HRE (Figure 1(b)). Therefore, it was indispensable to examine the resulting effects of an overexpression of mHSF1 on DCs, especially regarding (i) expression of phenotypic cell surface maturation markers, (ii) toxicity, and (iii) function.
3.2. Specific Induction of the Short $p H s p 70 B_{-29 /-242}^{\prime}$ Promoter in HeLa Cells. To develop a new and efficient dual promoterbased transcriptional DC-targeting strategy, we initially compared the activity of two different promoter fragments derived from the $5^{\prime}$ region of the human $h s p 70 B$ gene. In 1985, Richard Voellmy isolated and characterized a $450 \mathrm{bp}$ BamHI/HindIII fragment of a 70,000-dalton heat shock protein segment (Hsp70) involved in the control of transcription and translation of heat shock proteins [34]. Later, Schiller and colleagues reported this copy of the $h s p 70$ gene as the $h s p 70 B$ gene [35]. Four cis-acting heat shock regulatory sequences (HSE 1-4) were described, which were supposed to contribute to the Hsp70B promoter activity. Additionally, HSE 1-3 (within a short fragment of $\sim 200 \mathrm{bp}$ in the $5^{\prime}$ nontranscribed region) were shown to be sufficient for optimal activity in human HeLa cells. To determine if the shorter element is sufficient, we cloned a $-29 /-489 \mathrm{bp}$ and a $-29 /-242 \mathrm{bp}$ fragment of the human $h s p 70 B$ gene $5^{\prime}$ region (according to GenBank accession no. X13229) into the pGL3-Basic luciferase reporter vector resulting in $\mathrm{pHsp} 70 \mathrm{~B}_{-29 /-489}$ and pHsp70B $-29 /-242$, respectively (Figure 2(a)). Afterwards, plasmids were used to transfect HeLa cells. As a control we used the promoterless pGL3-Basic ("Basic") and the pGL3-SV40Promoter ("SV40") vector. The next day, transcriptional activity was induced by the transduction of HeLa cells with an adenovirus encoding for a mutant constitutively active HSF1 (Ad5mHSF1). Transduction with Ad5Luc1 or Mock treatment served as controls. Twenty-four hours later, a luciferase reporter assay was performed and results were normalized relative to the respective pGL3-SV40-Promoter control. As shown in Figure 2(b), the Hsp70B $-29 /-489$ and Hsp70B $-29 /-242$ promoter were exclusively induced by Ad5mHSF1 up to 16-fold relative to the SV40 promoter, whereas there was no induction in the Mock sample or by the control virus. Furthermore, neither Ad5Lucl nor Ad5mHSF1 influenced the transcriptional activity of the SV40 promoter and the Basic vector. Interestingly, there was no difference in the promoter activity when directly comparing the long $\mathrm{Hsp}_{30 \mathrm{~B}_{-29 /-489}}$ with the short Hsp70B $-29 /-242$ promoter, but looking closely at the Mock-treated versus the Ad5mHSF1-transduced cells for each promoter, we found an increase of up to 40 times for the longer promoter fragment and up to 64 times for the shorter one. Finally, the Hsp70B $-29 /-242$ promoter was listed as the Hsp70B' (HSPA6) promoter in the GenBank (accession no. NM_002155) in 2004.

Thus, we identified the Hsp70B $B_{-29 /-242}^{\prime}$ promoter to be highly and specifically induced in HeLa cells and for this reason it was used in the following experiments to generate the "modular promoter" system.

\subsection{Multiple Therapeutic Transgenes Can Be Simultaneously} Expressed under the Control of the Hsp70B ${ }_{-29 /-242}$ Promoter. In order to prove our concept that mHSF1 can induce not only the expression of single transgenes but also the simultaneous expression of several transgenes under the control of the Hsp70B ${ }_{-29 /-242}^{\prime}$ promoter, we generated plasmid vectors containing either a $\mathrm{Hsp} 70 \mathrm{~B}_{-29 /-242}^{\prime}$-MelA/ MART-1 (“pMelA”), a Hsp70B ${ }_{-29 /-242}$-BclxL (“pBclxL”), 


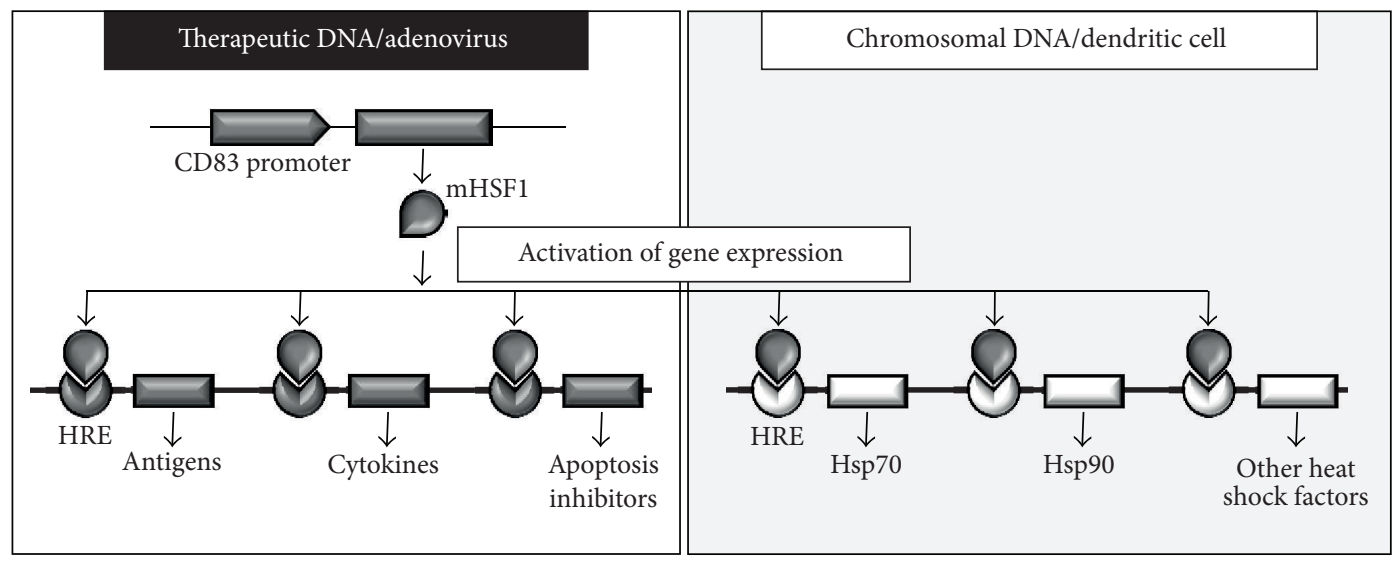

(a)

(b)

FIGURE 1: Schematic representation of the therapeutic DNA/adenovirus strategy and the effects of mHSF1 overexpression on endogenous heat shock response elements. (a) A constitutively active mutant heat shock factor 1 (mHSF1) will be expressed under the control of the human mDC-specific CD83 promoter. In turn mHSF1 binds to short heat shock response elements (HRE) of a second vector-encoded DNA driving expression of therapeutic transgenes (e.g., antigens, cytokines, or apoptosis inhibitors). (b) By overexpressing mHSF1 in the target cell, also endogenous HRE will become activated resulting in the expression of different heat shock proteins (Hsp), such as Hsp70 or Hsp90.

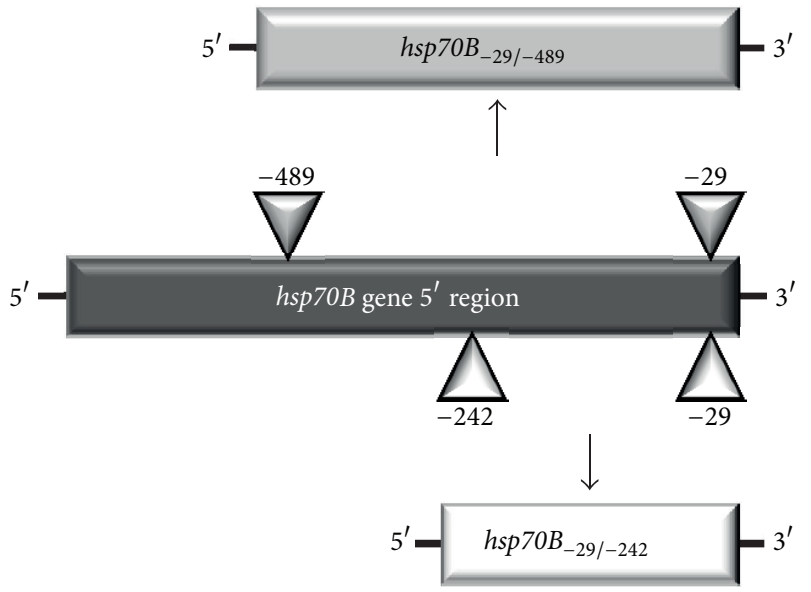

(a)

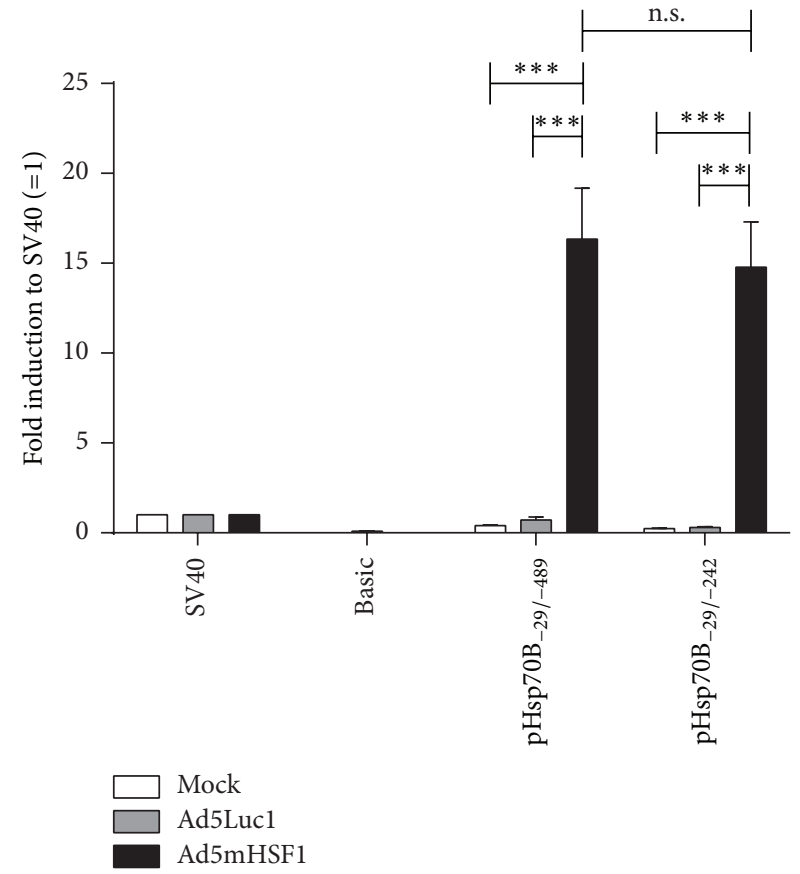

(b)

Figure 2: Generation and evaluation of two different Hsp70B heat shock response elements. (a) Plasmid vectors containing Hsp70B $\mathrm{B}_{-29 /-489}$ and $\mathrm{Hsp}_{70 \mathrm{~B}_{-29 /-242}}$ were generated by digesting the human $h s p 70 \mathrm{~B}$ gene $5^{\prime}$-region with HindIII/BamHI or HindIII/SmaI, respectively, followed by ligation into the pGL3Basic vector. (b) HeLa cells were transfected with $0.5 \mu \mathrm{g}$ of plasmid DNA of pGL3-SV40-promoter vector

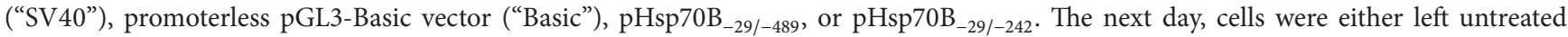
("Mock") or transduced with adenoviruses Ad5Lucl or Ad5mHSF1 at $300 \mathrm{TCID}_{50} /$ cell. Twenty-four hours later, cells were harvested and analyzed by luciferase reporter assays. Results are shown as fold induction relative to the individual pGL3-SV40-promoter control containing no virus, Ad5Lucl or Ad5mHSF1. Data are mean \pm SEM of three independent experiments. ${ }^{* * *} P<0.001$, n.s.: not significant $(P>0.05)$; bars without annotation are not significant $(P>0.05)$; two-way ANOVA with Bonferroni's Multiple Comparison post hoc test. 


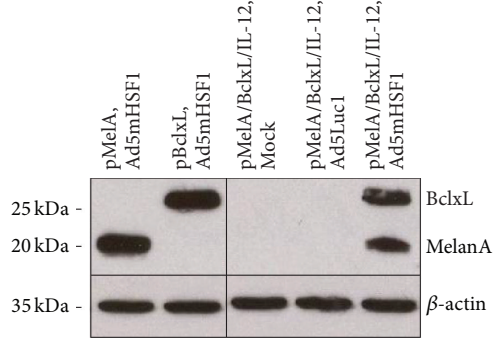

(a)

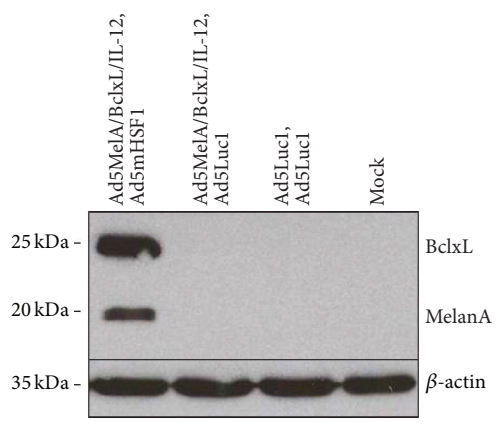

(c)
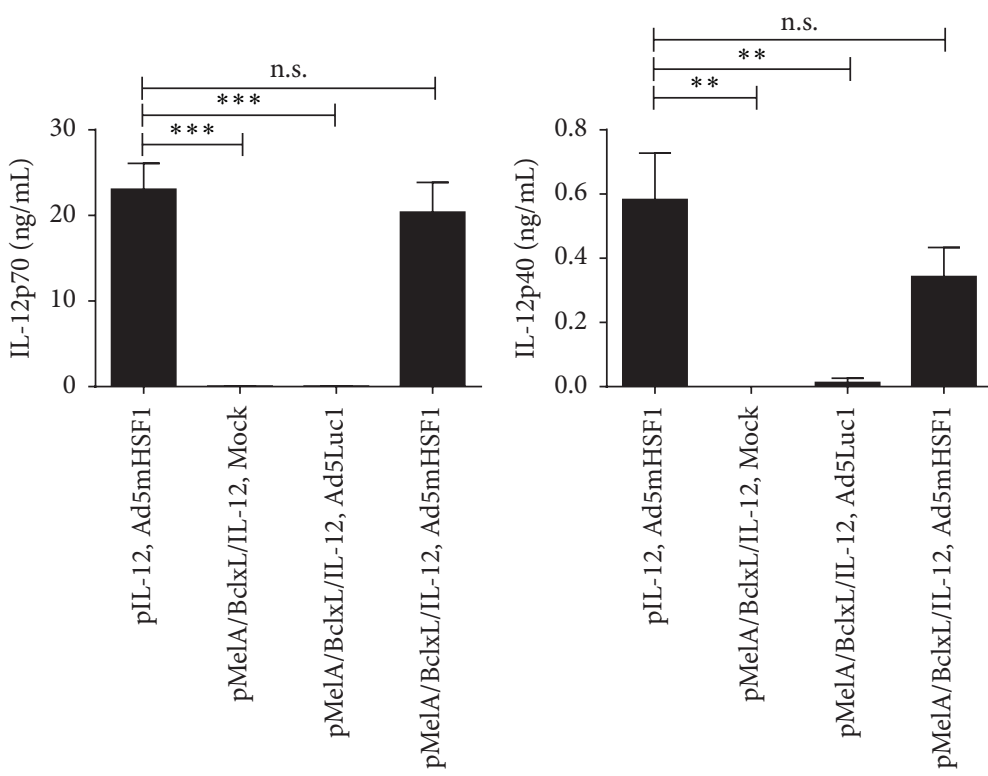

(b)
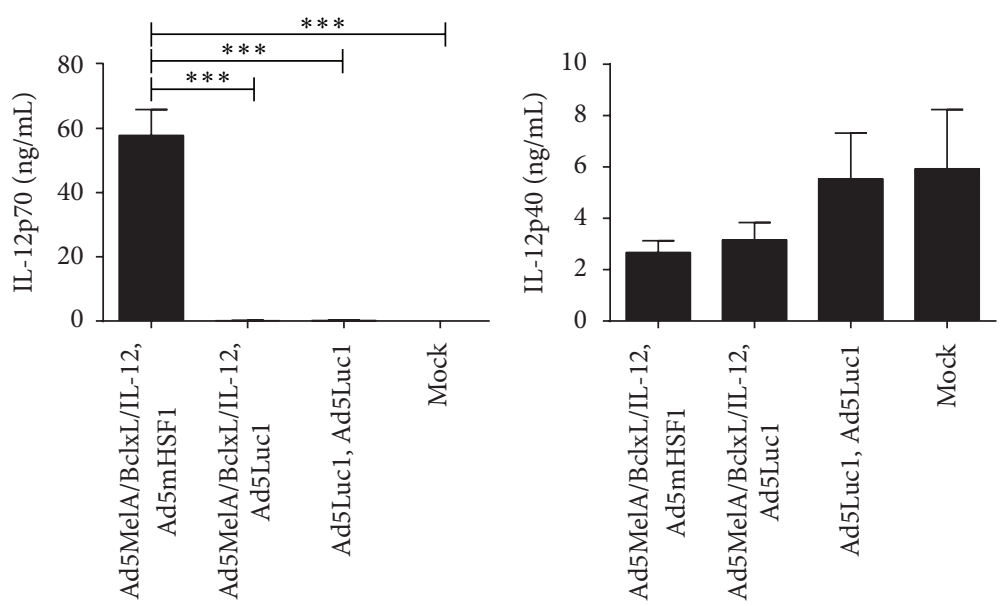

(d)

FIGURE 3: The constitutively active mHSF1 induces specific transgene expression in HeLa cells and human DCs. (a, b) HeLa cells were transfected with $0.7 \mu \mathrm{g}$ DNA of plasmid vectors expressing MelanA/MART-1 ("MelA"), BclxL or/and IL-12 under the control of the $\mathrm{Hsp} 70 \mathrm{~B}_{-29 /-242}^{\prime}$ heat shock response element as indicated. The next day, cells were either left untreated ("Mock") or transduced with adenoviruses Ad5Lucl or Ad5mHSF1 at 300 TCID $_{50} /$ cell. (c, d) Human immature DCs were cotransduced in the presence of the maturation cytokine cocktail with adenoviruses as indicated at a total amount of $500 \mathrm{TCID}_{50} /$ cell. (a-d) Twenty-four hours later, cell lysates were analyzed for BclxL and MelanA expression by Western Blot (a, c) and cell culture supernatants for content of IL-12p40 and IL-12p70 by ELISA (b, d). (a and c) Showing one representative experiment out of three; (b and d) data are mean \pm SEM of three independent experiments with different donors. ${ }^{* *} P<0.01,{ }^{* * *} P<0.001$, and n.s.: not significant $(P>0.05)$; bars without annotation are not significant $(P>0.05)$; one-way ANOVA with Bonferroni's Multiple Comparison post hoc test.

a Hsp70B' ${ }_{-29 /-242}$-IL-12 (“pIL-12”), or the triple Hsp70$\mathrm{B}_{-29 /-242}^{\prime}$-MelA-Hsp70B ${ }_{-29 /-242}$-BclxL-Hsp70B ${ }_{-29 /-242}$-IL-12 ("pMelA/BclxL/IL-12") expression cassette. HeLa cells were transfected with the above-mentioned vectors followed by transduction with Ad5mHSF1, Ad5Lucl, or Mock the next day. Twenty-four hours later cells were harvested, lysed, and separated by SDS-PAGE followed by Western Blotting using specific antibodies for MelanA/MART-1, BclxL, and beta-actin expression. As shown in Figure 3(a), MelanA and BclxL were highly expressed only after specific HSP70B' promoter activation by Ad5mHSF1. Of note, the MelanA and BclxL proteins were equally well expressed from the triple plasmid pMelA/BclxL/IL-12. For the determination of the IL-12 concentration in the cell culture supernatants of cells analyzed in (a), IL-12p70- and IL-12p40- specific ELISA were performed (Figure 3(b)). Again, IL-12 production after transfection with pIL-12 or pMelA/BclxL/IL-12 was induced by Ad5mHSF1. Transduced HeLa cells secreted high amounts of the immunologic active IL-12p70 $(23.06 \mathrm{ng} / \mathrm{mL}$ and $20.39 \mathrm{ng} / \mathrm{mL}$, resp.) and much lower amounts of the 
homodimer IL-12p40 (0.58 ng/mL and $0.34 \mathrm{ng} / \mathrm{mL}$, resp.). Moreover, there was almost no difference in the quantity of either IL-12p70 or IL-12p40 expressed from pIL-12 or pMelA/BclxL/IL-12, respectively.

Regarding our aim to insert the MP system into an adenoviral vector, we next generated Ad5MelA/BclxL/IL-

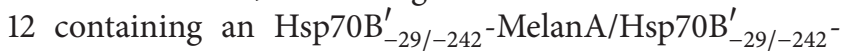
$\mathrm{BclxL} / \mathrm{Hsp} 70 \mathrm{~B}_{-29 /-242}^{\prime}$-IL-12 expression cassette. Adenovirus vectors contain several promoters within their own genome, for example, the early transcripts E1A-E4, as well as multiple recognition sites within these promoters for transcription factors. They often represent cis-acting DNA sequences that increase transcription, independent of their orientation and distance relative to the RNA start site [36]. To exclude the possible interference of these viral enhancers and upstream regulatory elements with the $\mathrm{Hsp} 70 \mathrm{~B}^{\prime}$ promoter of our system, we cotransduced human iDCs with Ad5MelA/BclxL/IL12, Ad5mHSF1 or control virus Ad5Lucl in the presence of a cytokine maturation cocktail. As a control, cells were matured in absence of an adenoviral vector ("Mock"). Twenty-four hours later, lysates of DCs were generated to perform Western Blot analyses to assess MelanA, BclxL, and beta-actin expression (Figure 3(c)). In addition, cell culture supernatants were analyzed by ELISA for the content of IL-12p70 and IL$12 \mathrm{p} 40$ (Figure $3(\mathrm{~d})$ ). As depicted there, only the cotransduction of Ad5MelA/BclxL/IL-12 with Ad5mHSF1 induced a specific transgene expression of MelanA, BclxL, and high amounts of secreted IL-12p70 (57.84 ng/mL). Furthermore, DCs transduced with Ad5MelA/BclxL/IL-12 produced less of the homodimer IL-12p40 in comparison to Mock or only Ad5Lucl treated DCs $(2.67$ and $3.16 \mathrm{ng} / \mathrm{mL}$ versus 5.93 and $5.54 \mathrm{ng} / \mathrm{mL}$, resp.).

In summary, we showed that the expression of multiple therapeutic transgenes under the control of the Hsp70B' promoter can specifically be induced in HeLa cells, as well as in primary human DCs. Moreover, we demonstrated that the Hsp70B' promoter acts in a highly specific manner, not only in a plasmid-based vector system, but particularly also in an adenoviral context.

3.4. Overexpression of $m H S F 1$ Induces Expression of Heat Shock Proteins and Does Not Impair DC Function. As overexpression of mHSF1 is also expected to induce the activation of endogenous heat shock proteins thereby inducing a heat shock response of Ad5mHSF1 treated DCs, we addressed the influence of mHSF1 overexpression on DCs as follows. Immature DCs were transduced with Ad5mHSF1 or control virus Ad5Lucl or Mock-treated either in the presence (mDC) or absence (iDC) of the maturation cocktail. Twenty-four and 48 hours later, analyses of DCs were conducted. First, regulation of heat shock proteins HSF1, Hsp40, Hsp70A, Hsp70B', Hsp90, and Grp94 was determined in cell lysates of DCs by SDS-PAGE followed by Western Blotting (Figure 4(a)). Here, a clear increase in expression of heat shock proteins Hsp40 and Hsp70A was observed for iDCs and mDCs, whereas Hsp70B' and Hsp90 was only induced in mDCs after Ad5mHSF1 treatment in comparison to Ad5Luc1 or Mock controls. HSF1 was only increased in Ad5Luc1 transduced mDCs, while mHSF1 was only found in DCs treated with Ad5mHSF1. Expression of Grp94 was unaffected by any treatment. Next, we examined the expression of typical cell surface activation markers including CD25, CD80, CD83, CD86, HLA-ABC (MHCI), and HLA-DR (MHCII) by FACS analyses (Figure 4(b)). As expected, all maturation markers of DCs treated with the cytokine cocktail ("mDC") for 24 as well as 48 hours were upregulated in comparison to iDCs. Notably, treatment with any type of adenoviral vectors did not induce maturation of iDCs when compared to the Mock control. Furthermore, when DCs were matured during transduction $(\mathrm{mDC})$, the adenoviral vectors did not influence the expression of the assessed surface markers. In addition to their activation status, DCs were also analyzed by FACS for their viability using propidium iodide (PI) staining. As shown in Figure 4(c), there was only a slight increase in the percentage of dead cells induced by the adenovirus treatment. The percentage of PI positive iDCs increased from $5.68 \%$ (Mock, 24h) and 7.10\% (Mock, 48 h) to 9.98\% (Ad5Lucl, $24 \mathrm{~h}$ ) and $11.90 \%$ (Ad5Lucl, $48 \mathrm{~h}$ ) or $13.20 \%$ (AdmHSF1, $24 \mathrm{~h}$ ) and $18.43 \%$ (AdmHSF1, $48 \mathrm{~h}$ ), respectively. Matured DCs remained almost uninfluenced when compared with noninfected cells $\left(2.20 \%\right.$ and $1.55 \% \mathrm{PI}^{+}$cells after $24 \mathrm{~h}$ or $48 \mathrm{~h}$, resp.). Transduction with Ad5Lucl resulted in $2.55 \%$ (24 h) or $1.78 \%(48 \mathrm{~h}$ ) and with Ad5mHSF1 in 4.68 (24h) or $5.13 \%$ (48 h) PI positive DCs.

Next, we assayed the functionality of these DCs by evaluating their capacity to secrete proinflammatory cytokines including IL-1 $\beta$, IL-6, IL-8, IL-12p70, and TNF- $\alpha$, as well as the anti-inflammatory cytokine IL-10, by cytometric bead array 24 and 48 hours after treatment (Figure $4(\mathrm{~d}))$. As the maturation cocktail contains IL-1 $\beta$, IL6 , and TNF- $\alpha$, Mock-treated DCs served as a control and yielded the background of the corresponding cytokine indicated by a red line in the graph. Interestingly, 24 and 48 hours after infection, Ad5mHSF1-transduced mDCs showed a superior secretion of IL-1 $\beta(2381 / 2451 \mathrm{pg} / \mathrm{mL})$, IL6 (13965/16740 pg/mL), IL-8 (41154/43674 pg/mL), IL-12p70 $(35 / 34 \mathrm{pg} / \mathrm{mL})$, and TNF- $\alpha(5176 / 5005 \mathrm{pg} / \mathrm{mL})$ to Mocktreated mDCs $(1443 / 1877 \mathrm{pg} / \mathrm{mL}, 8993 / 11063 \mathrm{pg} / \mathrm{mL}, 27581 /$ $32420 \mathrm{pg} / \mathrm{mL}, 10 / 7 \mathrm{pg} / \mathrm{mL}$, and $2757 / 1546 \mathrm{pg} / \mathrm{mL}$, resp.). This was not the case for Ad5Lucl transduced mDCs, where (with the exception of IL-12p70 and TNF- $\alpha$ ) only a slight increase of cytokine production for IL-1 $\beta$ (1710/1775 pg/mL), IL-6 $(11038 / 11519 \mathrm{pg} / \mathrm{mL})$, and IL-8 $(33398 / 34201 \mathrm{pg} / \mathrm{mL})$ could be observed either 24 or 48 hours after transduction. Interleukin-10 is only secreted at very low amounts but slightly enhanced at both time points in supernatants of Ad5mHSF1 (65/49 pg/mL) treated mDCs, but not of Ad5Luc1 $(37 / 24 \mathrm{pg} / \mathrm{mL})$ treated ones, in comparison to the Mock control $(34 / 24 \mathrm{pg} / \mathrm{mL})$. Immature DCs secreted almost no cytokines, independent of the treatment and time point at which they were analyzed. Finally, we assessed the functional ability of Ad5mHSF1 transduced mature DCs to prime autologous naïve $\mathrm{CD}^{+} \mathrm{T}$ cells. Therefore, iDCs were transduced with Ad5Lucl or Ad5mHSF1 or were Mock-treated in the presence of the cytokine maturation cocktail the day before they were transfected with MelanA RNA or peptide-loaded with MelanA. Subsequently, DCs were cocultured with MACS-sorted $\mathrm{CD}^{+} \mathrm{T}$ cells for seven days and afterwards 


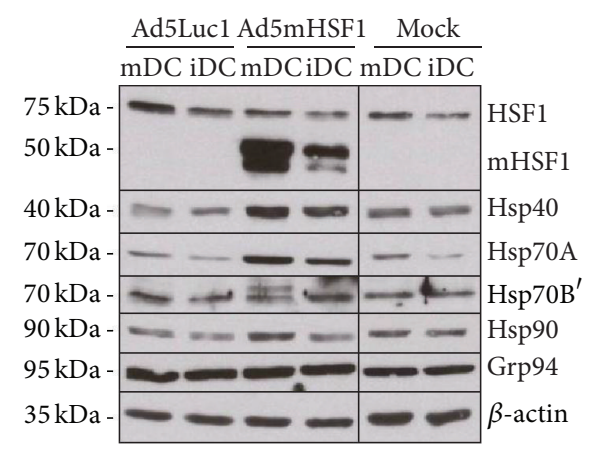

(a)
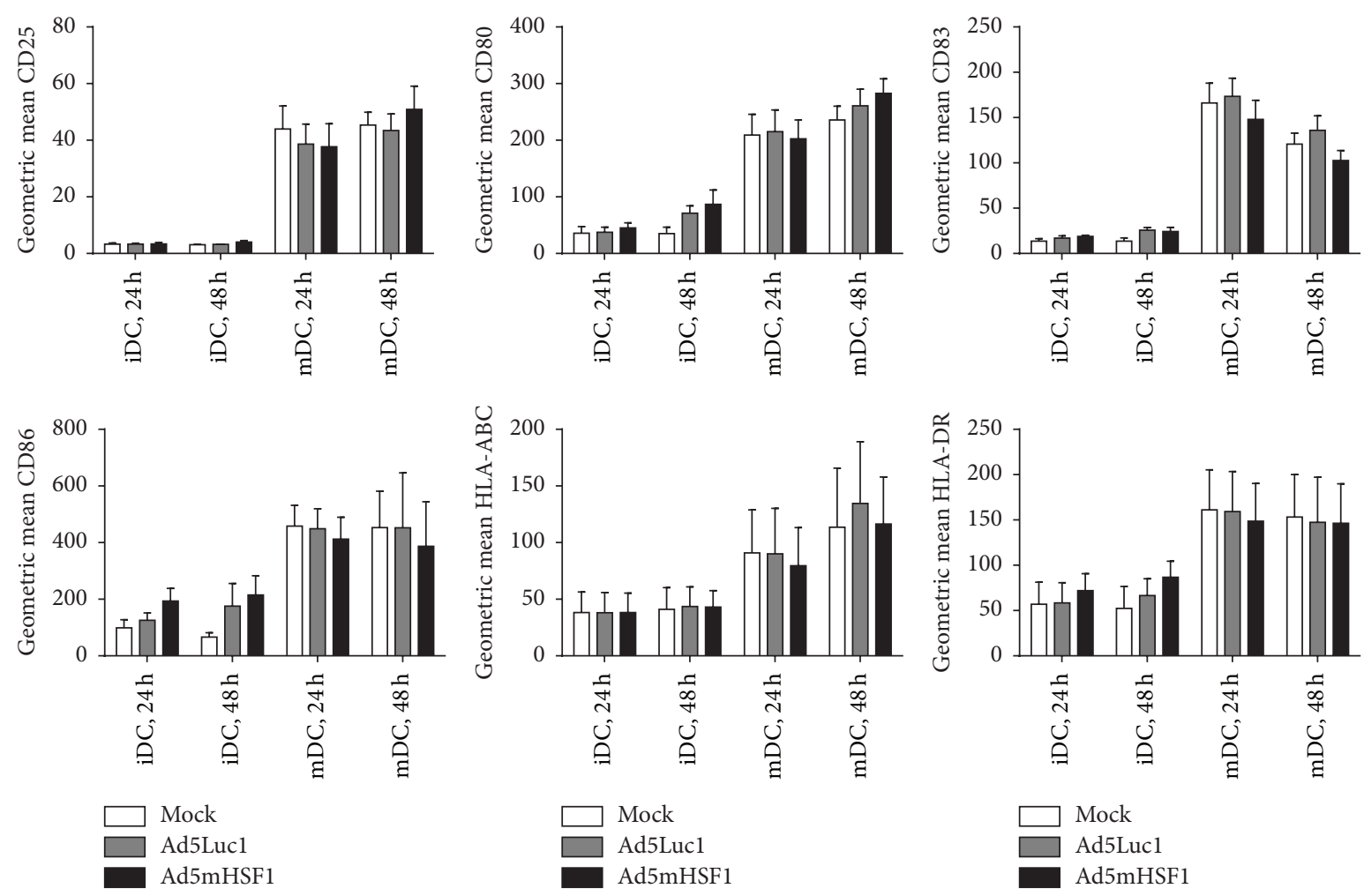

(b)
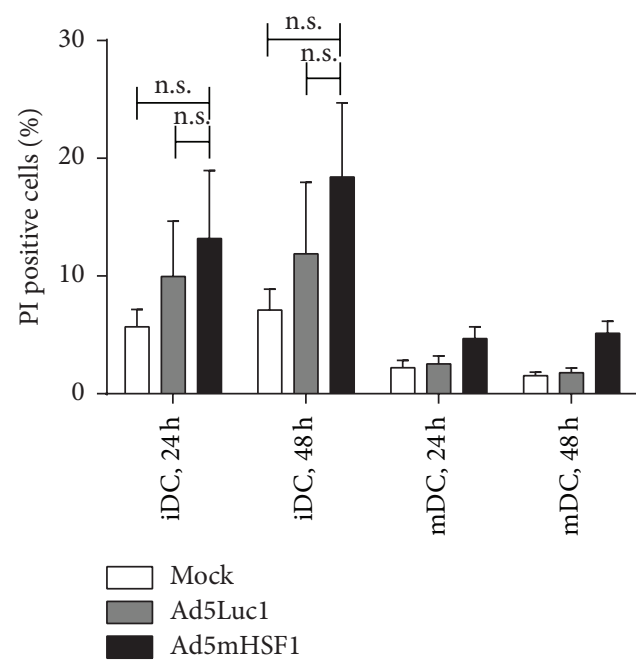

(c)

Figure 4: Continued. 

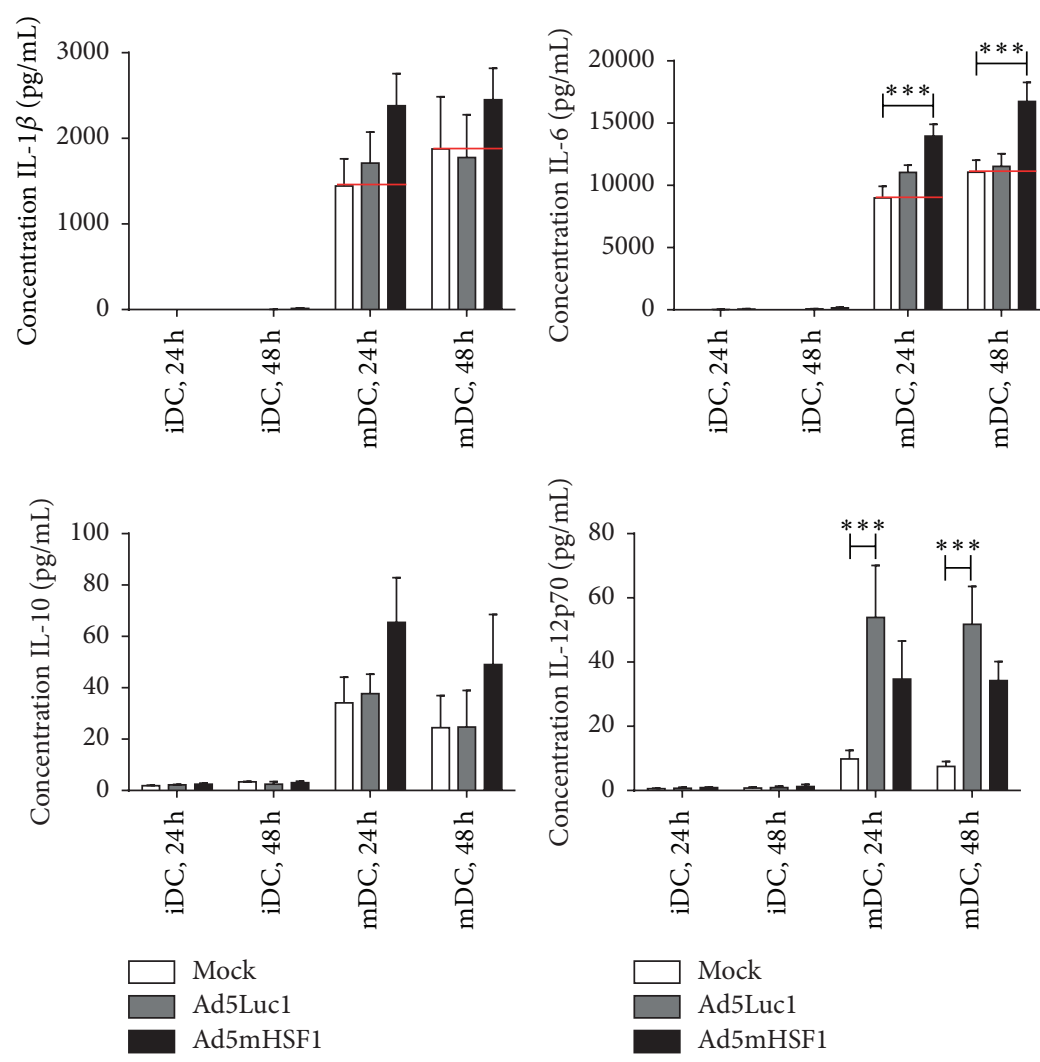

(d)
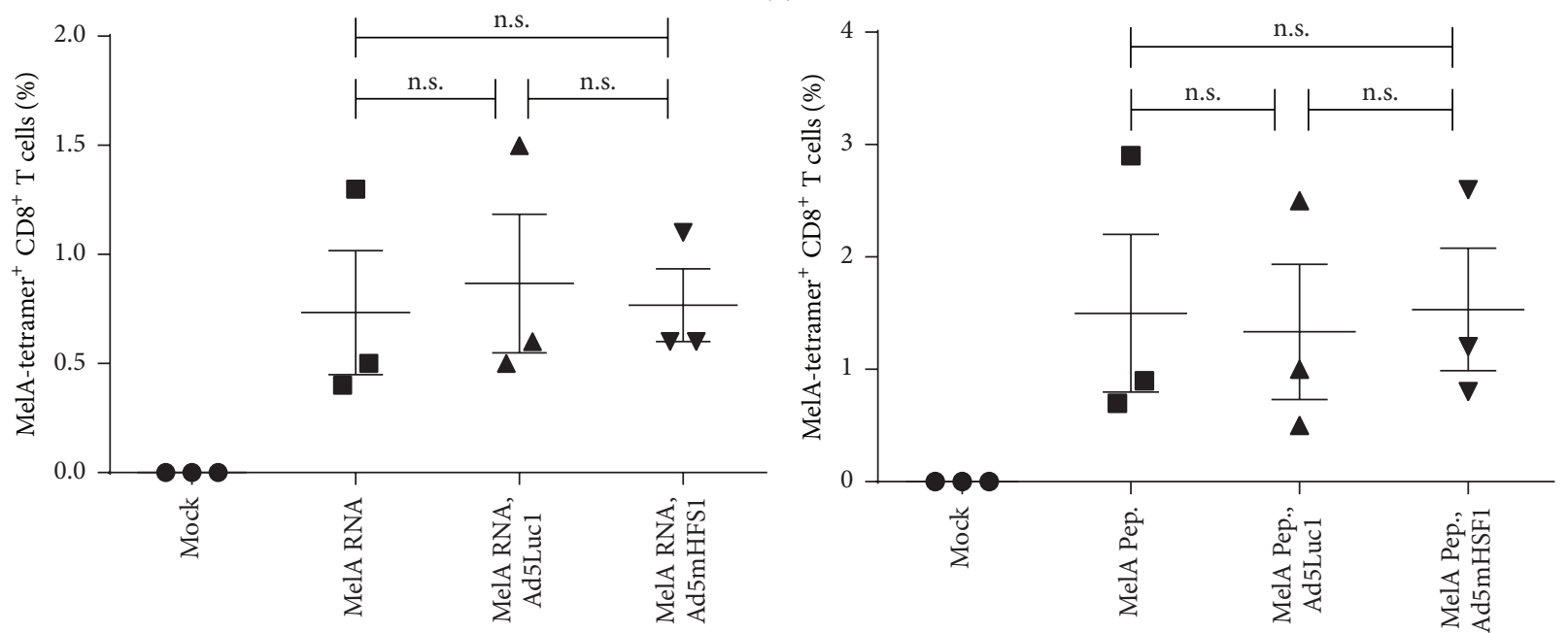

(e)

FIgURE 4: Overexpression of mHSF1 does not impair the phenotype and function of immature and mature DCs. Immature DCs were transduced with Ad5Lucl or Ad5mHSF1 at $500 \mathrm{TCID}_{50}$ /cell or Mock-treated and were either left immature ("iDC") or were matured (" $\mathrm{mDC}$ ") with a maturation cytokine cocktail (IL-1 $\beta$, IL-6, TNF- $\alpha$, and $\mathrm{PGE}_{2}$ ). (a) Western Blot analyses of cell lysates harvested $24 \mathrm{~h}$ after transduction on HSF1, mHSF1, Hsp40, Hsp70A, Hsp70B', Hsp90, Grp94, and beta-actin protein expression. One representative experiment out of three is shown. (b, c) Flow cytometric analyses of iDCs and mDCs on CD25, CD80, CD83, CD86, HLA-ABC, and HLA-DR (b) as well as PI (c) $24 \mathrm{~h}$ and $48 \mathrm{~h}$ after transduction. Data are mean \pm SEM of four independent experiments with DCs derived from different donors. (d) Cell culture supernatants derived from experiments shown in (b and c) were analyzed by cytometric bead array for the content of IL-1 $\beta$, IL-6, IL-8, IL-10, IL-12p70, and TNF- $\alpha$. The red line in (d) indicates background levels of respective cytokines derived from the cytokines present in the maturation cocktail. Data are mean \pm SEM of three independent experiments. (e) Twenty-four hours after adenoviral transduction accompanied by maturation of DCs, cells were either transfected with wild-type MelanA RNA (left panel) or loaded with MelanA analogue peptide (right panel). Antigen-loaded DCs were cocultured with MACS-sorted autologous CD ${ }^{+} \mathrm{T}$ cells for seven days. Induction of antigenspecific CTLs was determined by HLA-A2-MelanA/iTag MHC class I-tetramer and anti-CD8-PC7 staining using a FC500 cytofluorometer. Data are mean \pm SEM of three independent experiments with different donors. (b-e) ${ }^{*} P<0.05,{ }^{* *} P<0.01$, ${ }^{* * *} P<0.001$, and n.s.: not significant $(P>0.05)$; bars without annotation are not significant $(P>0.05)$, one-way, (e) or two-way ANOVA (b-d) with Bonferroni's Multiple Comparison post hoc test. 
analyzed by staining with a HLA-A2-MelanA/iTag MHC class I-tetramer and an anti-CD8 antibody followed by flow cytometry. As shown in Figure 4(e), only antigen-loaded DCs induced the proliferation of MelanA-specific $\mathrm{CD}^{+} \mathrm{T}$ cells with analogue peptide-loaded DCs (1.5\%) being twofold more potent than wild-type RNA-transfected $(0.73 \%)$ DCs. Moreover, transduction with neither the control adenovirus (RNA: $0.87 \%$, Pep: $1.34 \%$ ) nor Ad-mediated overexpression of mHSF1 (RNA: $0.77 \%$, Pep: $1.53 \%$ ) impaired the capacity of antigen-loaded DCs to induce specific CTL responses, independent of the route of delivery.

From these data we conclude that neither transduction with the used adenoviral vectors per se nor the overexpression of mHSF1 interfered with the function of DCs. However, transduction with Ad5mHSF1 resulted in the upregulation of various heat shock proteins and proinflammatory cytokines by mDCs.

3.5. Specific Targeting of Mature DCs by a Combined CD83Hsp $70 B^{\prime}$ Promoter System. To realize our final goal of transcriptionally targeting human mature DCs, we created new vectors where the expression of mHSF1 is driven by the human CD83 promoter, recently characterized by our group [18]. This tripartite promoter complex, consisting of a $510 \mathrm{bp}$ upstream plus core promoter element ("P-510") and a $185 \mathrm{bp}$ enhancer ("E") fragment, was shown to act in a cell typeand maturation-specific manner. To assess this, also in the context of the modular promoter system, we generated plasmid vectors (Figure 5(a)) containing mHSF1 and only the P-510 CD83 promoter ("pP-510/mHSF1") or the P-510 plus the enhancer in either the sense or antisense direction ("pEs/P-510/mHSF1" or "pEas/P-510/mHSF1"). These were cotransfected into the murine DC-like cell line XS52 with plasmid vectors expressing MelanA/MART-1 and IL12 under the control of the Hsp70B ${ }_{-29 /-242}^{\prime}$ HRE ("pMP2"; Figures 5(a)-5(c)). As a control pMP2 was replaced by (i) a vector comprising only the Hsp70B ${ }_{-29 /-242}^{\prime}$ promoter or (ii) the empty pLG3-Basic vector ("Basic") (Figures 5(a) and 5(b)). Twenty-four hours later, cells were lysed for SDSPAGE and Western Blotting for MelanA and beta-actin detection, while cell culture supernatants were analyzed by ELISA for the content of IL-12p70 and IL-12p40. As depicted in Figure 5(b), only cotransfection of pMP2 with pEs/P$510 / \mathrm{mHSF} 1$ or $\mathrm{pEas} / \mathrm{P}-510 / \mathrm{mHSF} 1$ resulted in a strong and robust MelanA expression, while pP-510/mHSF1 without the enhancer showed only weak transgene expression. Accordingly, IL-12p70 secretion was not detectable in controls, at low levels in the context of pMP2 plus pP-510/mHSF1 transfected cells $(1.07 \mathrm{ng} / \mathrm{mL})$ and in large amounts by the combination of pMP2 with $\mathrm{pEs} / \mathrm{P}-510 / \mathrm{mHSF} 1(4.08 \mathrm{ng} / \mathrm{mL})$ or pEas/P-510/mHSF1 $(4.79 \mathrm{ng} / \mathrm{mL}$ ) (Figure 5(c)). Interleukin$12 \mathrm{p} 40$, however, was only detectable in supernatants derived from $\mathrm{pMP} 2 \mathrm{plus} \mathrm{pEs} / \mathrm{P}-510 / \mathrm{mHSF} 1(0.01 \mathrm{ng} / \mathrm{mL})$ or $\mathrm{pEas} / \mathrm{P}-$ $510 / \mathrm{mHSF} 1(0.434 \mathrm{ng} / \mathrm{mL})$ transfected XS52 cells.

Finally, we generated corresponding adenoviral vectors, that is, Ad5MP2, Ad5P-510/mHSF1, Ad5Es/P-510/mHSF1, and Ad5Eas/P-510/mHSF1. These were used together with Ad5Lucl (negative control), Ad5MelA (positive control), or Ad5mHSF1 to cotransduce immature human DCs. DCs were then either left immature ("iDC") or were matured with LPS for 20 hours ("mDC") because this maturation stimulus induced the highest expression of the CD83 promoter ([18] and unpublished results). Afterwards, cell lysates were analyzed for expression of MelanA and betaactin by Western Blot and supernatants by IL-12p70 and IL-12p40 ELISA. Clearly, MelanA expression was specifically induced only in mDCs after cotransduction of Ad5MP2 with adenoviruses containing the CD83 promoter-mHSF1 cassette (Figure 5(d)). Positive controls Ad5MP2 plus Ad5mHSF1, as well as Ad5MelA plus Ad5Lucl, revealed expression of the transgene in iDCs and $\mathrm{mDCs}$, while negative controls did not show a signal for either. Similar results were obtained for cytokine-matured DCs (data not shown). Regarding IL-12p70, LPS-matured DCs, in contrast to cocktail-matured DCs, produced higher amounts in general (Figure 5(e)). Interestingly, in comparison to controls, that is, Ad5MP2 plus Ad5Luc1 $(19.05 \mathrm{ng} / \mathrm{mL})$ or Ad5MelA plus Ad5Luc1 $(16.1 \mathrm{ng} / \mathrm{mL})$, the amount of IL-12p70 expressed doubled when Ad5MP2 was cotransduced with Ad5P-510/mHSF1 (35.87 ng/mL), Ad5Es/P-510/mHSF1 (41.46 ng/mL), or Ad5Eas/P-510/mHSF1 (57.56 ng/mL). Cocktail-matured DC (data not shown) showed a lower, but specific, IL-12p70 production upon cotransduction of Ad5MP2 with Ad5P-510/ mHSF1 (9.83 ng/mL), Ad5Es/P-510/mHSF1 (16.78 ng/mL), or Ad5Eas/P-510/mHSF1 (16.83 ng/mL). In comparison to LPSmatured DCs, immature DCs showed a lower expression of IL-12p70 (max. $8.93 \mathrm{ng} / \mathrm{mL}$ ), while in the positive control (Ad5MP2 plus Ad5mHSF1), higher amounts of IL-12p70 were detected for both LPS-matured $(52.3 \mathrm{ng} / \mathrm{mL})$ and immature DCs $(80.96 \mathrm{ng} / \mathrm{mL})$. In contrast, IL-12p40 was mainly secreted by control-transduced LPS-matured DCs (max. $29.54 \mathrm{ng} / \mathrm{mL}$ ), whereas LPS-matured DCs transduced with the MP adenoviruses showed less (max. $13.05 \mathrm{ng} / \mathrm{mL}$ ) and iDCs no IL-12p40 irrespective of the adenovirus used.

Taken together, we have here demonstrated our modular promoter system to specifically target human immunogenic mature DCs to efficiently induce the expression of different therapeutic transgenes.

\section{Discussion}

One big challenge facing cancer immunotherapy is the generation of cell-specific treatment strategies. For in vivo DC-targeting, the restriction of transgene expression to this specific cell type is the main interest. Adenoviruses display several advantages for this targeted delivery of therapeutic transgenes such as tumor antigens and immune stimulatory proteins. They permit an extended expression of full length antigens within the transduced DC. In contrast to other targeting strategies, where peptides, proteins, or mRNAs are used, the cancer-proteins are synthesized over a longer period and processed by the DCs' own antigen-presentation machinery. As a consequence, long-lasting antigen-presentation to $\mathrm{T}$ cells is ensured without generating concerns regarding the breakdown of peptide/MHC complexes [37].

Melanoma is viewed as an "immunogenic" tumor type which is why here Ad-based and other strategies to improve 


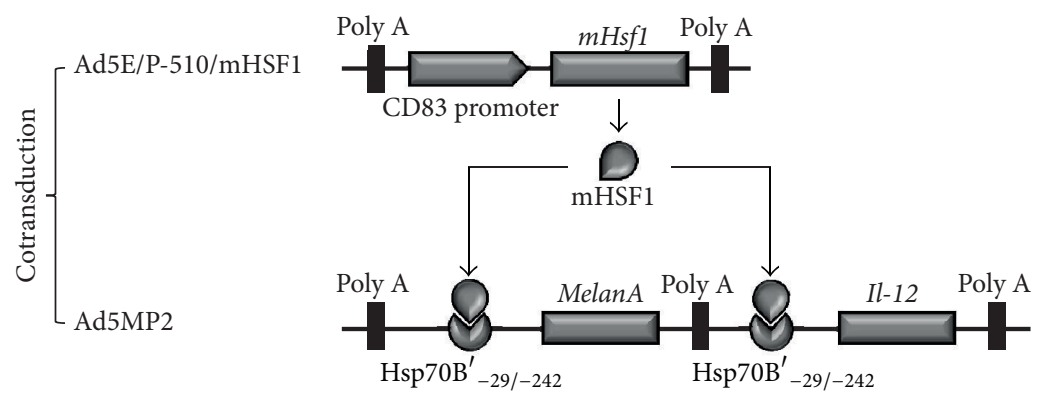

(a)

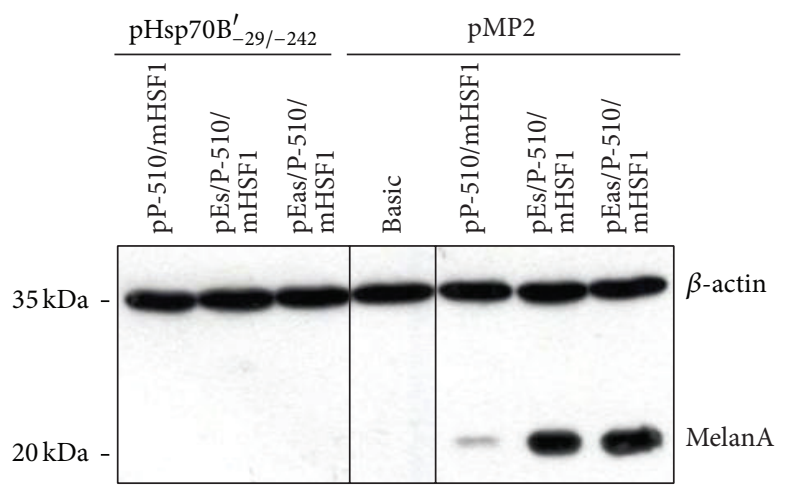

(b)
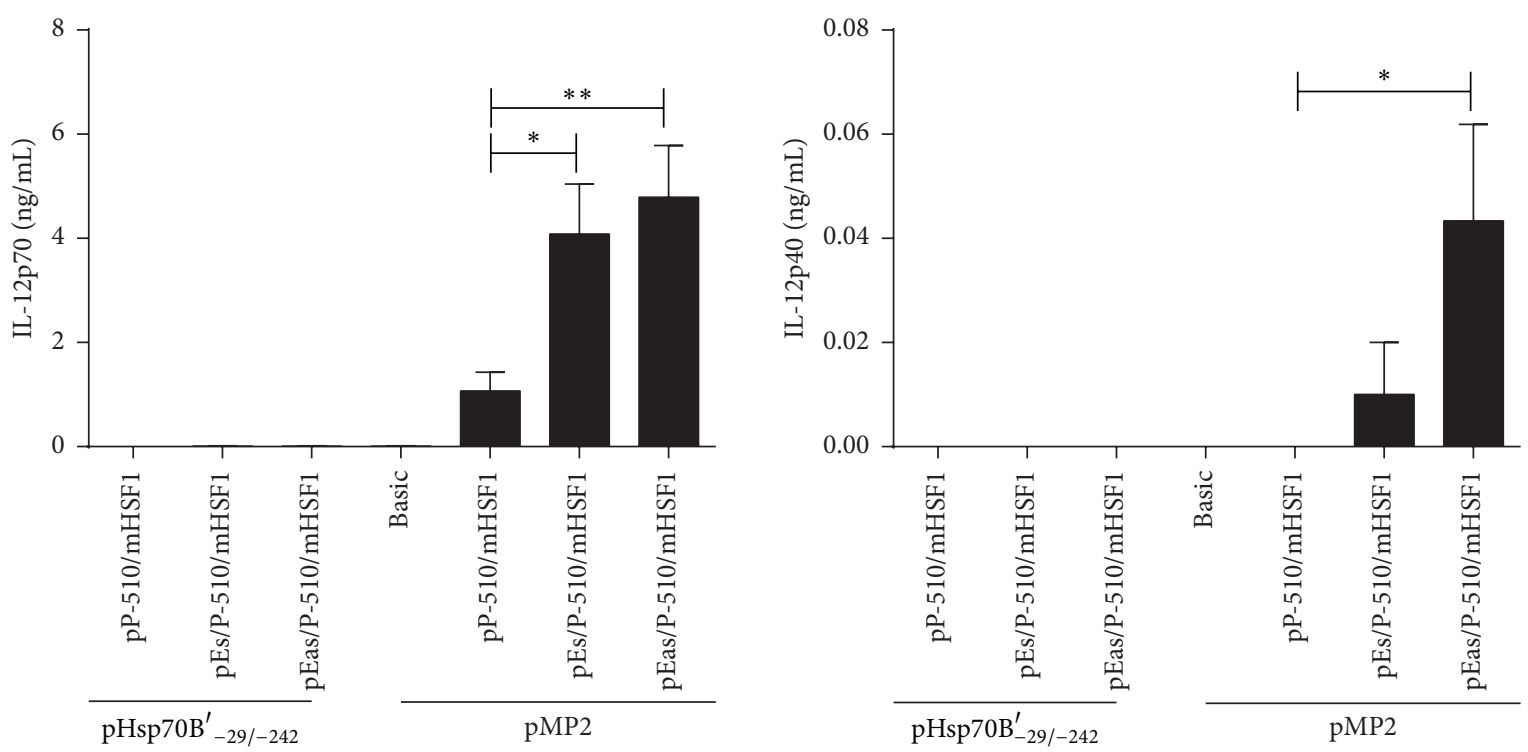

(c)

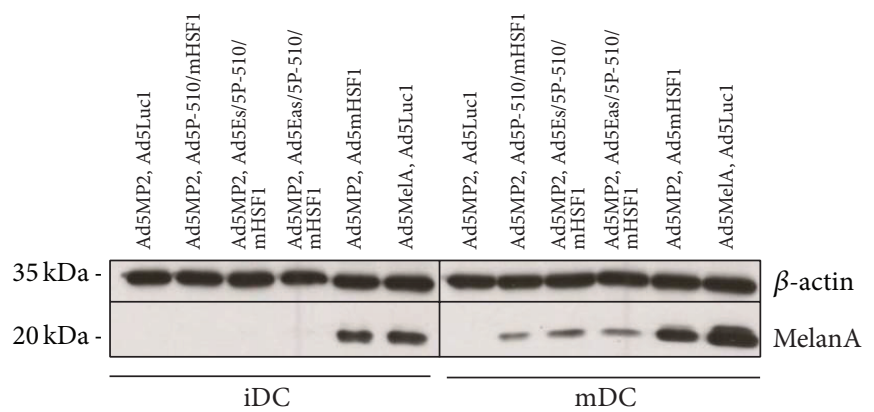

(d)

FIgUre 5: Continued. 


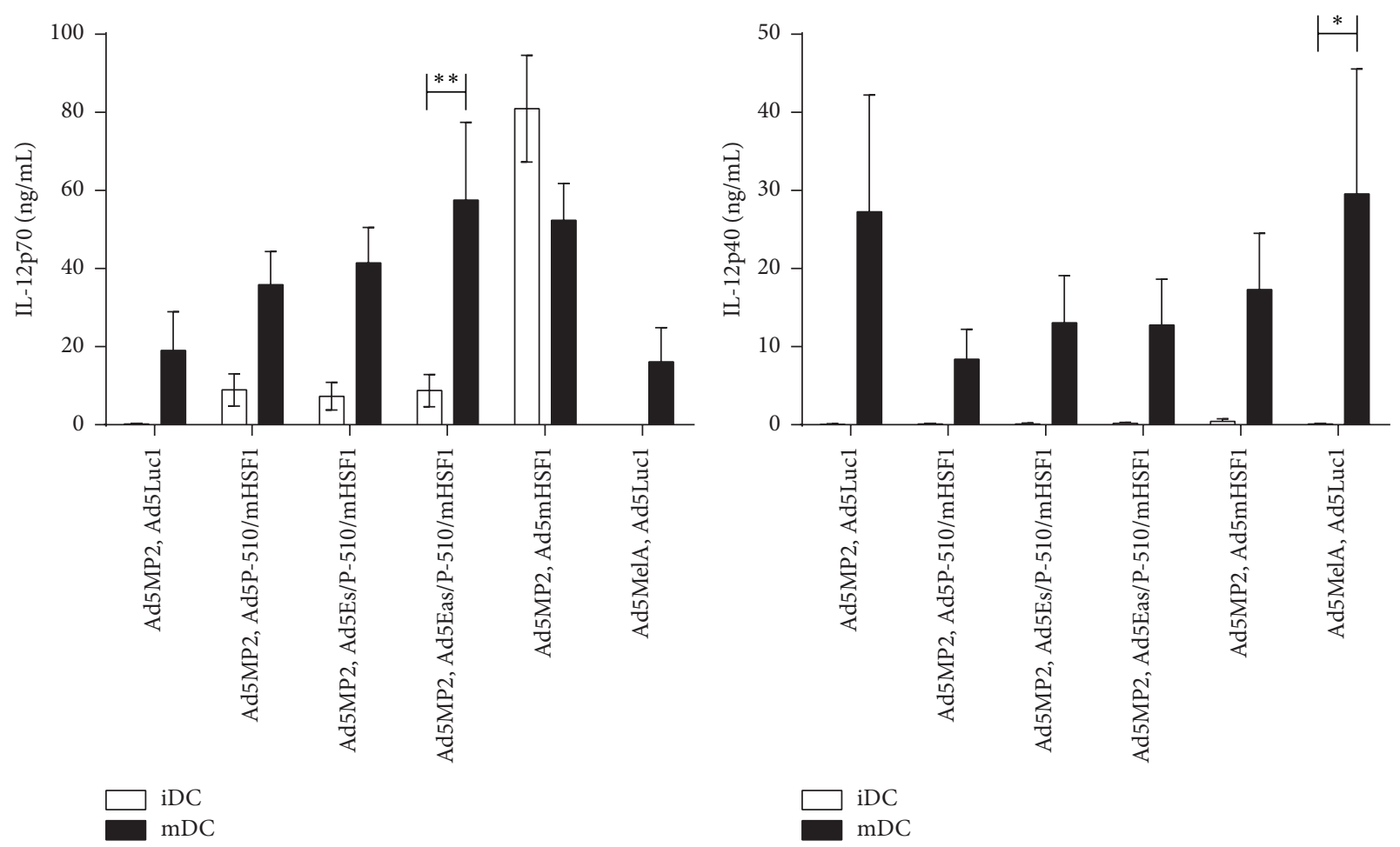

(e)

FIGURE 5: The "modular promoter (MP)" system enables highly efficient and specific expression of therapeutic transgenes in mature DCs. (a) Schematic outline of the dual vector "modular promoter" system. Ad5E/P-510/mHSF1 drives expression of mHSF1 under the control of the cell type- and maturation-specific human CD83 promoter, which in turn induces specific induction of therapeutic transgenes MelanA and Il-12 by binding to the Hsp70B ${ }_{-29 /-242}^{\prime}$ HRE encoded by Ad5MP2. (b, c) XS52 cells were cotransfected with a total amount of $2 \mu \mathrm{g}$ of plasmid vectors pP-510/mHSF1, pEs/P-510/mHSF1, or pEas/P-510/mHSF1 in combination with plasmid vectors encoding the HRE alone ("pHsp70B' or the modular promoter system ("pMP2"). As a control, the empty pGL3-Basic vector ("Basic") was used. (d, e) Human immature DCs were cotransduced with Ad5MP2 or Ad5MelA in combination with Ad5Luc1, Ad5mHSF1, Ad5P-510/mHSF1, Ad5Es/P-510/mHSF1, or Ad5Eas/P510/mHSF1. Cells were either left immature or were matured by adding $0.1 \mathrm{ng} / \mathrm{mL}$ LPS. (b-e) Twenty hours after transfection, cell lysates were analyzed by Western Blot for expression of MelanA and beta-actin (b, d) and cell culture supernatants by ELISA for content of IL-12p40 and IL-12p70 (c, e). Either one representative $(b, d)$ or data mean \pm SEM from three independent experiments $(c, e)$ is shown. ${ }^{*} P<0.05$; ${ }^{* *} P<0.01$; bars without annotation are not significant $(P>0.05)$, one-way ANOVA (c) or two-way ANOVA (e) with Bonferroni’s Multiple Comparison post hoc test.

DC-mediated immunotherapies have been used for the treatment of this disease. Moreover, malignant melanoma is the most common cause of mortality from skin cancer worldwide. It is refractory to irradiation and chemotherapy in late phases but rather amenable to immunological approaches [38]. Although great efforts have been made in recent years, antitumor DC-based vaccines rarely exceeded $15 \%$ of the objective clinical responses [39]. Hence, there is still a high medical need to develop new strategies to improve DC vaccination.

In this study, we present in vitro experiments using a combined CD83-Hsp70B' promoter system ("modular promoter [MP]" system; Figures 1 and 5(a)) to specifically target mature DCs for the simultaneous expression of different therapeutic transgenes in the context of malignant melanoma. Therefore, we expressed a mutated HSF1 (mHSF1) with a deletion between amino acid positions 202-316 of wild-type HSF1, which has been shown to be constitutively active even in the absence of stress [26], under the control of the DCand maturation-specific human CD83 promoter [18]. In turn, mHSF1 is used to drive simultaneous $\mathrm{Hsp} 70 \mathrm{~B}^{\prime}$-dependent therapeutic transgene expression. Heat shock promoters, particularly Hsp70 promoters, have often been used for gene therapy in the past and the $\mathrm{Hsp} 70 \mathrm{~B}^{\prime}$ promoter displays several advantages. First, it is induced only after severe stress, resulting in a lower basal expression compared to other $h s p 70$ genes [40,41]. This is in accordance with our data, as these demonstrated the $\mathrm{Hsp} 70 \mathrm{~B}^{\prime}$ promoter to be induced only by mHSF1 and not to be sensitive to stress factors induced by lipofection (Figure 2(b)) or adenoviral transduction (Figure 3). Second, the moderate expression of $\mathrm{Hsp} 70 \mathrm{~B}^{\prime}$ was reported to be restricted to white blood cells like DCs, monocytes, and NK cells especially but to be nearly absent in other blood cells and tissues [42]. Third, the core $\mathrm{Hsp} 70 \mathrm{~B}^{\prime}$ promoter element is very short ( $213 \mathrm{bp}$ ) and showed a promoter activity 16 times higher than the strong 
SV40 promoter (Figure 2(b)). In addition, (m)HSF1 is not immunogenic as it is an ubiquitously expressed protein.

Interestingly, overexpression of $\mathrm{mHSF} 1 \mathrm{did}$ not influence the maturation status, the survival of immature and mature DCs, or their capability to prime naïve $\mathrm{CD}^{+} \mathrm{T}$ cells in an antigen-specific manner whereas heat treatment of these DCs did result in the upregulation of maturation markers and increased capability to prime naïve $\mathrm{CD}^{+} \mathrm{T}$ cells [27]. However, mHSF1 overexpression or adenoviral transduction of $\mathrm{mDCs}$ per se induced elevated levels of proinflammatory cytokines including IL- $1 \beta$, IL-6, IL-8, IL-12p70, and TNF- $\alpha$ in cell culture supernatants. We also found Hsp40 and Hsp70A to be upregulated in cell lysates of iDCs and mDCs, whereas Hsp70B' and Hsp90 were only induced in mDCs. Heat shock proteins such as Hsp40 and Hsp70 have been described to act as chaperones and be involved in the proper folding of proteins as well as in the prevention of the formation of nascent protein aggregations thereby keeping the proteins in a substrate-grabbable state. Others, like Hsp90, have been demonstrated to contribute to antigen-presentation on MHC I [43-45]. In this regard, heat shock proteins also became the focus of attention in the vaccine research area. Exogenous Hsp70 and Hsp90 have been reported to induce secretion of cytokines like GM-CSF, IL- $1 \beta$, IL- 6 , IL12 , and TNF- $\alpha$ and to upregulate cell surface maturation markers CD83, CD86, or CD40 as well as to enhance the migratory capacity of DCs [46-48]. Induction of heat shock proteins, on the other hand, is ensued by the induction of HSF1 [49]. Besides its role as a transcription factor in stressed cells, HSF1 is involved in a multitude of physiological processes such as cell metabolism, gametogenesis, aging, insulin signaling, and cancer progression $[24,25]$. Regarding the latter, HSF1 was shown to act on cancer development by regulating tumor cell proliferation, antiapoptosis, epithelialmesenchymal transition (EMT), migration, invasion, and metastasis [50]. Interestingly, almost nothing is known about the influence of HSF1 or mHSF1 overexpression on human DCs. Thus, at least to our knowledge, this is the first study indicating that overexpression of mHSF1 does not impair DC maturation and function, although varying reports on the DC's responsiveness to heat shock were published [27, 51-53]. Solely Ostberg and colleagues reviewed that effects such as the induction of maturation or stimulation of T cells, induced by mild thermal stress, are not dependent on HSF1-mediated transcriptional events in murine bone marrow derived DCs [54]. The mHSF1 used in the present study however was also overexpressed by Xia and colleagues in HeLa cells [55]. As a consequence, HeLa cells, which are normally relatively insensitive to Fas-mediated killing, became sensitive to Fasinduced apoptosis, thereby providing a new target for Fasbased antitumor vaccines.

Encouraged by these results, we generated the modular promoter system to transcriptionally target mature DCs. To perform first proof of principle, we successfully expressed several therapeutic transgenes under the control of the core Hsp70B' promoter element, which we could specifically induce with CMV promoter-driven mHSF1 both in HeLa cells by plasmid transfection and in primary mature human DCs by adenoviral vectors (Figure 3 ). In a second step, we developed a two-vector-based system, where we set mHSF1 under the control of the human DC- and maturationspecific CD83 promoter to express MelanA and IL-12p70 (Figure 5(a)). The advantage of MelanA is its widespread expression on melanocytic cells and its ubiquitin-mediated proteasome-dependent degradation leading to the presentation of the antigen via MHC class I [56]. Interleukin-12p70, on the other hand, enhances the cytotoxic activity of $\mathrm{CD}^{+}$ CTLs and NK cells [57]. Moreover, exogenous expression of IL-12p70 is able to overcome the inhibitory effect of $\mathrm{PGE}_{2}$ on IL-12p70 production of DCs matured with the standard cytokine maturation cocktail used not only in vitro but also in vivo in clinical trials [58]. Of note, the combined CD83-Hsp70B' promoter system not only facilitated specific transcriptional targeting of mature immunogenic DCs but also guaranteed high expression of the respective transgenes (Figure 5) without affecting endogenous CD83 expression in maturing DCs (data not shown).

To date, only very few promoter systems have been proven suitable for the transcriptional targeting of DCs. The group of Ross et al., for instance, demonstrated the promoter of the cytoskeletal fascin protein to induce specific transgene expression in mature murine DCs after gene gun-mediated DNA immunization [59]. Similar results were also reported by Sudowe et al. to induce type 1 immune responses [59, $60]$. In the context of a lentiviral vector system, Dresch and colleagues described the $5^{\prime}$ untranslated region from the $D C$ STAMP gene as a suitable promoter region to yield longterm and cell-selective transgene expression in murine DCs in vivo [61]. Several cell type-specific promoters have been reported in the past to target murine DCs in vitro and in vivo; however here we report the first promoter system allowing the specific targeting of mature human DCs. Regarding its future prospects for cancer therapy, the CD83-Hsp70B ${ }^{\prime}$ promoter system offers several interesting features. Due to its modular composition, the system is highly flexible and hence not restricted to a specific tumor entity or therapeutic application. On the one hand, the therapeutic transgenes encoded by one adenoviral vector could be replaced by any gene of interest, even mutated cancer- and patient-specific TAAs. On the other hand, it would be possible to use an even more specific promoter or one with different cell-specificity, for the expression of the mHSF1 within the other vector. This modular composition would in theory allow the in vivo targeting of any combination of transgenes to any cell type although this requires substantial additional preparatory experiments. Moreover, by combining transductional targeting of adenoviruses to dendritic cells via bispecific diabodies or single-chain antibodies incorporated into the virus capsid, a further increase of targeting specificity might be achieved.

Finally, since CD83 is not expressed by immature but is highly induced in mature DCs, the applied CD83 promoter complex represents an ideal candidate for transcriptional targeting of mature DCs in vivo and could set the stage for next generation in situ vaccination strategies. This should be particularly effective and safe, as it assures selective antigen expression in $\mathrm{mDCs}$ while avoiding expression in tolerogenic iDCs for the first time. These promising perspectives, 
however, need to first be analyzed and explored in further preclinical investigations.

\section{Conclusions}

In this study, we present the initial in vitro experiments showing that a combined two-vector CD83-Hsp70B' promoter system is able to transcriptionally target human mature DCs for the first time with a highly specific and effective expression of a panel of potential therapeutic transgenes. Moreover, we first demonstrate that overexpression of mHSF1 does not interfere with DC maturation, viability, and function. Hence, this study provides valuable new insights for the development of a safe, modular, highly flexible, and hence patient-tailored vaccination protocol for in vivo targeting of DCs, but also other efficient genetic therapies in the future.

\section{Competing Interests}

The authors declare that there are no competing interests regarding the publication of this paper.

\section{Acknowledgments}

This work was supported by the Deutsche Forschungsgemeinschaft (DFG) via the CRC643 (Projects B13 and C1) and the ELAN-Fonds of the Universitätsklinikum Erlangen. The authors thank A. Takashima (University of Texas Southwestern Medical Center, TX, US), F. Schnieders (Provecs Medical GmbH, Hamburg, Germany), R. Voellmy (HSF Pharmaceuticals, Fribourg, Switzerland), and D. T. Curiel (Washington University School of Medicine, MO, US) for kindly providing research material and Vera E. Assmann (Universitätsklinikum Erlangen, Erlangen, Germany) for proofreading the paper.

\section{References}

[1] J. Datta, E. Berk, J. A. Cintolo, S. Xu, R. E. Roses, and B. J. Czerniecki, "Rationale for a multimodality strategy to enhance the efficacy of dendritic cell-based cancer immunotherapy," Frontiers in Immunology, vol. 6, article 271, 2015.

[2] I. Caminschi and K. Shortman, "Boosting antibody responses by targeting antigens to dendritic cells," Trends in Immunology, vol. 33, no. 2, pp. 71-77, 2012.

[3] W. R. Heath and F. R. Carbone, "Cross-presentation, dendritic cells, tolerance and immunity," Annual Review of Immunology, vol. 19, pp. 47-64, 2001.

[4] N. Bloy, J. Pol, F. Aranda et al., "Trial watch: dendritic cell-based anticancer therapy," OncoImmunology, vol. 3, no. 11, Article ID e963424, 2014.

[5] A. Makkouk and G. J. Weiner, "Cancer immunotherapy and breaking immune tolerance: new approaches to an old challenge," Cancer Research, vol. 75, no. 1, pp. 5-10, 2015.

[6] K. Palucka and J. Banchereau, "Dendritic-cell-based therapeutic cancer vaccines," Immunity, vol. 39, no. 1, pp. 38-48, 2013.

[7] J. Constantino, C. Gomes, A. Falcão, M. T. Cruz, and B. M. Neves, "Antitumor dendritic cell-based vaccines: lessons from
20 years of clinical trials and future perspectives," Translational Research, vol. 168, pp. 74-95, 2016.

[8] R. M. Steinman, "Decisions about dendritic cells: past, present, and future," Annual Review of Immunology, vol. 30, pp. 1-22, 2012.

[9] G. Schreibelt, L. J. J. Klinkenberg, L. J. Cruz et al., "The C-type lectin receptor CLEC9A mediates antigen uptake and (cross-) presentation by human blood BDCA3+ myeloid dendritic cells," Blood, vol. 119, no. 10, pp. 2284-2292, 2012.

[10] V. Apostolopoulos, T. Thalhammer, A. G. Tzakos, and L. Stojanovska, "Targeting antigens to dendritic cell receptors for vaccine development," Journal of Drug Delivery, vol. 2013, Article ID 869718, 22 pages, 2013.

[11] P. J. Tacken, I. S. Zeelenberg, L. J. Cruz et al., "Targeted delivery of TLR ligands to human and mouse dendritic cells strongly enhances adjuvanticity," Blood, vol. 118, no. 26, pp. 6836-6844, 2011.

[12] A. Garu, G. Moku, S. K. Gulla, and A. Chaudhuri, "Genetic immunization with in vivo dendritic cell-targeting liposomal DNA vaccine carrier induces long-lasting antitumor immune response," Molecular Therapy, vol. 24, no. 2, pp. 385-397, 2015.

[13] L. J. Cruz, P. J. Tacken, I. S. Zeelenberg et al., "Tracking targeted bimodal nanovaccines: immune responses and routing in cells, tissue, and whole organism," Molecular Pharmaceutics, vol. 11, no. 12, pp. 4299-4313, 2014.

[14] R. Hernandez-Alcoceba, J. Poutou, M. C. Ballesteros-Briones, and C. Smerdou, "Gene therapy approaches against cancer using in vivo and ex vivo gene transfer of interleukin-12," Immunotherapy, vol. 8, no. 2, pp. 179-198, 2016.

[15] R. A. A. Oldham, E. M. Berinstein, and J. A. Medin, "Lentiviral vectors in cancer immunotherapy," Immunotherapy, vol. 7, no. 3, pp. 271-284, 2015.

[16] W. S. M. Wold and K. Toth, "Adenovirus vectors for gene therapy, vaccination and cancer gene therapy," Current Gene Therapy, vol. 13, no. 6, pp. 421-433, 2013.

[17] S. D. Barker, I. P. Dmitriev, D. M. Nettelbeck et al., "Combined transcriptional and transductional targeting improves the specificity and efficacy of adenoviral gene delivery to ovarian carcinoma," Gene Therapy, vol. 10, no. 14, pp. 1198-1204, 2003.

[18] M. F. Stein, S. Lang, T. H. Winkler et al., "Multiple interferon regulatory factor and NF- $\kappa \mathrm{B}$ sites cooperate in mediating celltype-and maturation-specific activation of the human CD83 promoter in dendritic cells," Molecular and Cellular Biology, vol. 33, no. 7, pp. 1331-1344, 2013.

[19] L. Vandenberk, J. Belmans, M. Van Woensel, M. Riva, and S. W. Van Gool, "Exploiting the immunogenic potential of cancer cells for improved dendritic cell vaccines," Frontiers in Immunology, vol. 6, article 663, 2016.

[20] S. Rohmer, A. Mainka, I. Knippertz, A. Hesse, and D. M. Nettelbeck, "Insulated hsp70B' promoter: stringent heat-inducible activity in replication-deficient, but not replication-competent adenoviruses," Journal of Gene Medicine, vol. 10, no. 4, pp. 340354, 2008.

[21] C. Rome, F. Couillaud, and C. T. W. Moonen, "Spatial and temporal control of expression of therapeutic genes using heat shock protein promoters," Methods, vol. 35, no. 2, pp. 188-198, 2005.

[22] R. Voellmy, "Transduction of the stress signal and mechanisms of transcriptional regulation of heat shock/stress protein gene expression in higher eukaryotes," Critical Reviews in Eukaryotic Gene Expression, vol. 4, no. 4, pp. 357-401, 1995. 
[23] R. Baler, G. Dahl, and R. Voellmy, "Activation of human heat shock genes is accompanied by oligomerization, modification, and rapid translocation of heat shock transcription factor HSF1," Molecular and Cellular Biology, vol. 13, no. 4, pp. 24862496, 1993.

[24] A. Vihervaara and L. Sistonen, "HSF1 at a glance," Journal of Cell Science, vol. 127, no. 2, pp. 261-266, 2014.

[25] J. Anckar and L. Sistonen, "Regulation of HSF1 function in the heat stress response: implications in aging and disease," Annual Review of Biochemistry, vol. 80, pp. 1089-1115, 2011.

[26] J. Zuo, D. Rungger, and R. Voellmy, "Multiple layers of regulation of human heat shock transcription factor 1," Molecular and Cellular Biology, vol. 15, no. 8, pp. 4319-4330, 1995.

[27] I. Knippertz, M. F. Stein, J. Dörrie et al., "Mild hyperthermia enhances human monocyte-derived dendritic cell functions and offers potential for applications in vaccination strategies," International Journal of Hyperthermia, vol. 27, no. 6, pp. 591603, 2011.

[28] I. A. Pfeiffer, E. Zinser, E. Strasser et al., "Leukoreduction system chambers are an efficient, valid, and economic source of functional monocyte-derived dendritic cells and lymphocytes," Immunobiology, vol. 218, no. 11, pp. 1392-1401, 2013.

[29] N. Schaft, J. Dörrie, I. Müller et al., "A new way to generate cytolytic tumor-specific T cells: electroporation of RNA coding for a T cell receptor into T lymphocytes," Cancer Immunology, Immunotherapy, vol. 55, no. 9, pp. 1132-1141, 2006.

[30] R. Waehler, H. Ittrich, L. Mueller, G. Krupski, D. Ameis, and F. Schnieders, "Low-dose adenoviral immunotherapy of rat hepatocellular carcinoma using single-chain interleukin-12," Human Gene Therapy, vol. 16, no. 3, pp. 307-317, 2005.

[31] T. C. He, "Adenoviral vectors," in Current Protocols in Human Genetics, chapter 12, John Wiley \& Sons, New York, NY, USA, 2004.

[32] X. Liao, Y. Li, C. Bonini et al., "Transfection of RNA encoding tumor antigens following maturation of dendritic cells leads to prolonged presentation of antigen and the generation of highaffinity tumor-reactive cytotoxic T lymphocytes," Molecular Therapy, vol. 9, no. 5, pp. 757-764, 2004.

[33] N. Schaft, V. Wellner, C. Wohn, G. Schuler, and J. Dörrie, "CD8 T-cell priming and boosting: More antigen-presenting DC, or more antigen per DC?" Cancer Immunology, Immunotherapy, vol. 62, no. 12, pp. 1769-1780, 2013.

[34] R. Voellmy, A. Ahmed, P. Schiller, P. Bromley, and D. Rungger, "Isolation and functional analysis of a human 70,000-dalton heat shock protein gene segment," Proceedings of the National Academy of Sciences of the United States of America, vol. 82, no. 15, pp. 4949-4953, 1985.

[35] P. Schiller, J. Amin, J. Ananthan, M. E. Brown, W. A. Scott, and R. Voellmy, "Cis-acting elements involved in the regulated expression of a human hsp70 gene," Journal of Molecular Biology, vol. 203, no. 1, pp. 97-105, 1988.

[36] D. S. Steinwaerder and A. Lieber, "Insulation from viral transcriptional regulatory elements improves inducible transgene expression from adenovirus vectors in vitro and in vivo," Gene Therapy, vol. 7, no. 7, pp. 556-567, 2000.

[37] L. H. Butterfield and L. Vujanovic, "New approaches to the development of adenoviral dendritic cell vaccines in melanoma," Current Opinion in Investigational Drugs, vol. 11, no. 12, pp. 1399-1408, 2010.

[38] S. Bramante, J. K. Kaufmann, V. Veckman et al., "Treatment of melanoma with a serotype $5 / 3$ chimeric oncolytic adenovirus coding for GM-CSF: results in vitro, in rodents and in humans," International Journal of Cancer, vol. 137, no. 7, pp. 1775-1783, 2015.

[39] S. Anguille, E. L. Smits, E. Lion, V. F. Van Tendeloo, and Z. N. Berneman, "Clinical use of dendritic cells for cancer therapy," The Lancet Oncology, vol. 15, no. 7, pp. e257-e267, 2014.

[40] T. K. C. Leung, M. Y. Rajendran, C. Monfries, C. Hall, and L. Lim, "The human heat-shock protein family: expression of a novel heat-inducible HSP70 (HSP70B') and isolation of its cDNA and genomic DNA," Biochemical Journal, vol. 267, no. 1, pp. 125-132, 1990.

[41] K.-I. Wada, A. Taniguchi, and T. Okano, "Highly sensitive detection of cytotoxicity using a modified HSP70B' promoter," Biotechnology and Bioengineering, vol. 97, no. 4, pp. 871-876, 2007.

[42] M. Daugaard, M. Rohde, and M. Jäättelä, “The heat shock protein 70 family: highly homologous proteins with overlapping and distinct functions," The FEBS Letters, vol. 581, no. 19, pp. 3702-3710, 2007.

[43] T. Ichiyanagi, T. Imai, C. Kajiwara et al., "Essential role of endogenous heat shock protein 90 of dendritic cells in antigen cross-presentation," The Journal of Immunology, vol. 185, no. 5, pp. 2693-2700, 2010.

[44] T. Imai, Y. Kato, C. Kajiwara et al., "Heat shock protein 90 (HSP90) contributes to cytosolic translocation of extracellular antigen for cross-presentation by dendritic cells," Proceedings of the National Academy of Sciences of the United States of America, vol. 108, no. 39, pp. 16363-16368, 2011.

[45] Y. Kato, C. Kajiwara, I. Ishige et al., "HSP70 and HSP90 differentially regulate translocation of extracellular antigen to the cytosol for cross-presentation," Autoimmune Diseases, vol. 2012, Article ID 745962, 10 pages, 2012.

[46] S. Basu, R. J. Binder, R. Suto, K. M. Anderson, and P. K. Srivastava, "Necrotic but not apoptotic cell death releases heat shock proteins, which deliver a partial maturation signal to dendritic cells and activate the NF- $\kappa$ B pathway," International Immunology, vol. 12, no. 11, pp. 1539-1546, 2000.

[47] D. G. Millar, K. M. Garza, B. Odermatt et al., "Hsp70 promotes antigen-presenting cell function and converts T-cell tolerance to autoimmunity in vivo," Nature Medicine, vol. 9, no. 12, pp. 1469-1476, 2003.

[48] R. P. A. Wallin, A. Lundqvist, S. H. Moré, A. Von Bonin, R. Kiessling, and H.-G. Ljunggren, "Heat-shock proteins as activators of the innate immune system," Trends in Immunology, vol. 23, no. 3, pp. 130-135, 2002.

[49] N. Hentze, L. Le Breton, J. Wiesner, G. Kempf, and M. P. Mayer, "Molecular mechanism of thermosensory function of human heat shock transcription factor Hsf1," eLife, vol. 5, article e11576, 2016.

[50] S. Jiang, K. Tu, Q. Fu et al., "Multifaceted roles of HSF1 in cancer," Tumor Biology, vol. 36, no. 7, pp. 4923-4931, 2015.

[51] S. Basu and P. K. Srivastava, "Fever-like temperature induces maturation of dendritic cells through induction of hsp90," International Immunology, vol. 15, no. 9, pp. 1053-1061, 2003.

[52] K. Matsumoto, N. Yamamoto, S. Hagiwara et al., "Optimization of hyperthermia and dendritic cell immunotherapy for squamous cell carcinoma," Oncology Reports, vol. 25, no. 6, pp. 15251532, 2011.

[53] A. Semmlinger, M. Fliesser, A. M. Waaga-Gasser et al., "Fever-range temperature modulates activation and function of human dendritic cells stimulated with the pathogenic mould 
Aspergillus fumigatus," Medical Mycology, vol. 52, no. 4, pp. 438-444, 2014.

[54] J. R. Ostberg and E. A. Repasky, "Emerging evidence indicates that physiologically relevant thermal stress regulates dendritic cell function," Cancer Immunology, Immunotherapy, vol. 55, no. 3, pp. 292-298, 2006.

[55] W. Xia, R. Voellmy, and N. L. Spector, "Sensitization of tumor cells to fas killing through overexpression of heat-shock transcription factor 1," Journal of Cellular Physiology, vol. 183, no. 3, pp. $425-431,2000$.

[56] C. Setz, M. Friedrich, S. Hahn et al., "Just one positionindependent lysine residue can direct MelanA into proteasomal degradation following N-terminal fusion of ubiquitin," PLoS ONE, vol. 8, no. 2, Article ID e55567, 2013.

[57] A. L. Croxford, P. Kulig, and B. Becher, "IL-12-and IL-23 in health and disease," Cytokine and Growth Factor Reviews, vol. 25, no. 4, pp. 415-421, 2014.

[58] M. Erdmann and B. Schuler-Thurner, "Dendritic cell vaccines in metastasized malignant melanoma," Giornale Italiano di Dermatologia e Venereologia, vol. 143, no. 4, pp. 235-250, 2008.

[59] R. Ross, S. Sudowe, J. Beisner et al., “Transcriptional targeting of dendritic cells for gene therapy using the promoter of the cytoskeletal protein fascin," Gene Therapy, vol. 10, no. 12, pp. 1035-1040, 2003.

[60] S. Sudowe, I. Ludwig-Portugall, E. Montermann, R. Ross, and A. B. Reske-Kunz, "Transcriptional targeting of dendritic cells in gene gun-mediated DNA immunization favors the induction of type 1 immune responses," Molecular Therapy, vol. 8, no. 4, pp. 567-575, 2003.

[61] C. Dresch, S. L. Edelmann, P. Marconi, and T. Brocker, "Lentiviral-mediated transcriptional targeting of dendritic cells for induction of T cell tolerance in vivo," Journal of Immunology, vol. 181, no. 7, pp. 4495-4506, 2008. 


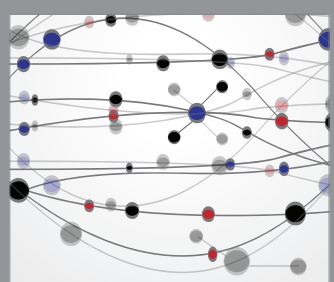

The Scientific World Journal
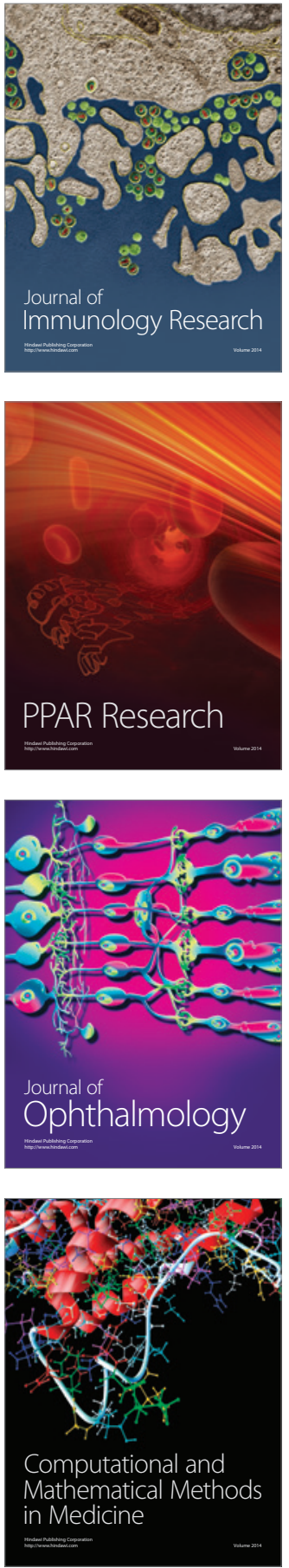

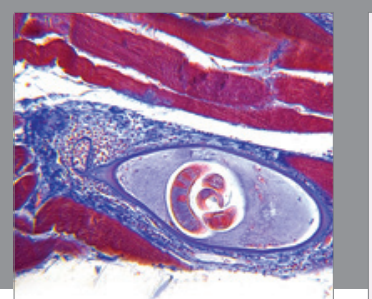

Gastroenterology Research and Practice

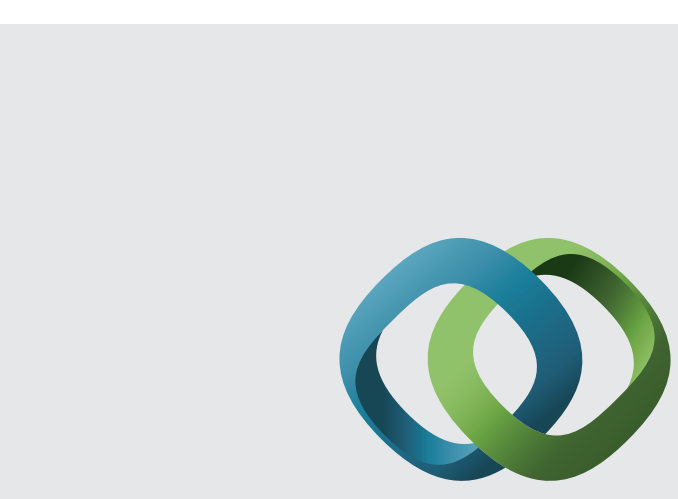

\section{Hindawi}

Submit your manuscripts at

http://www.hindawi.com
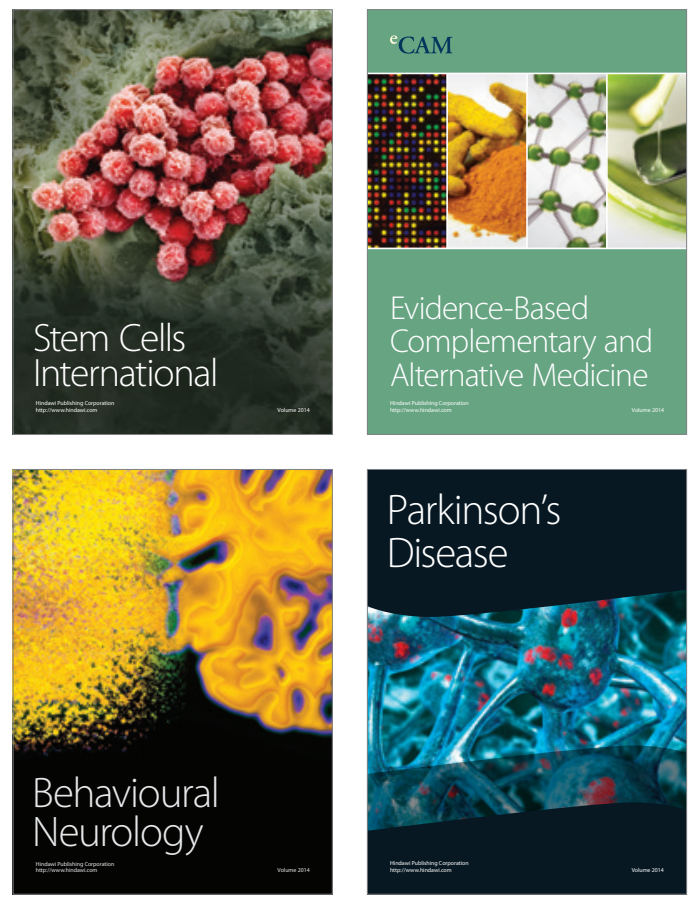
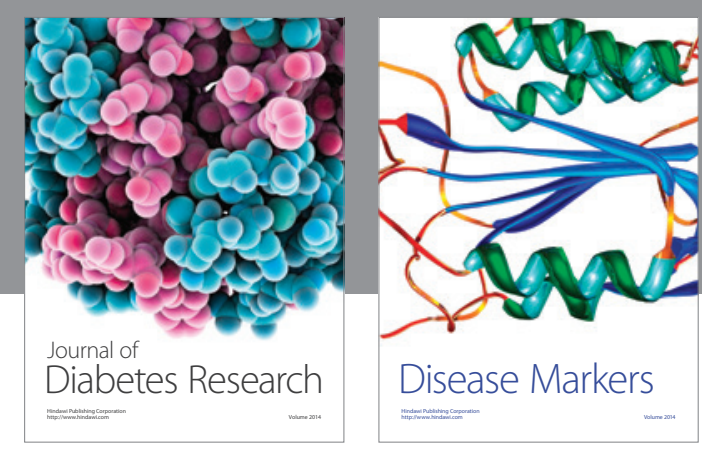

Disease Markers
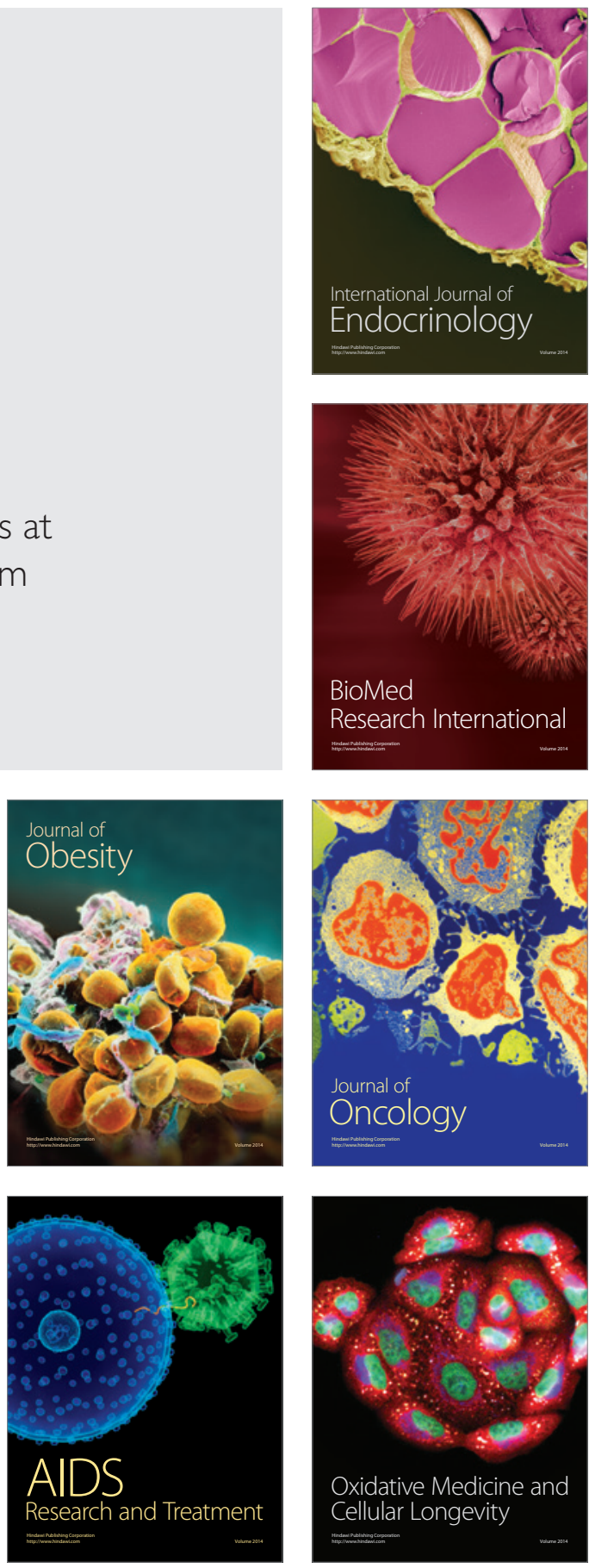